\title{
Interaction of bimodal fields with few-level atoms in cavities and traps
}

\author{
A. Messina, S. Maniscalco and A. Napoli \\ INFM, MURST and Dipartimento di Scienze Fisiche ed Astronomiche, \\ via Archirafi 36, 90123 Palermo, Italy \\ Tel/Fax: +39 91 6234243; E-mail: messina@fisica.unipa.it
}

February 1, 2008

\begin{abstract}
The spectacular experimental results of the last few years in cavity quantum electrodynamics and trapped ions research has led to very high level laboratory performances. Such a stimulating situation essentially stems from two decisive advancements. The first is the invention of reliable protocols for the manipulation of single atoms. The second is the ability to produce desired bosonic environments on demand. These progresses have led to the possibility of controlling the form of the coupling between individual atoms and an arbitrary number of bosonic modes. As a consequence, fundamental matter-radiation interaction models like, for instance, the JC model and most of its numerous nonlinear multiphoton generalizations, have been realized or simulated in laboratory and their dynamical features have been tested more or less in detail. This topical paper reviews the state of the art of the theoretical investigations and of the experimental observations concerning the dynamical features of the coupling between single few-level atoms and two bosonic modes. In the course of the paper we show that such a configuration provides an excellent platform for investigating various quantum intermode correlation effects tested or testable in the cavity quantum electrodynamics and trapped ion experimental realms. In particular we discuss a mode-mode correlation effect appearing in the dynamics of a two-level atom quadratically coupled to two bosonic modes. This effect, named parity effect, consists in a high sensitivity to the evenness or oddness of the total number of bosonic excitations.
\end{abstract}




\section{Contents}

1 Foreword 3

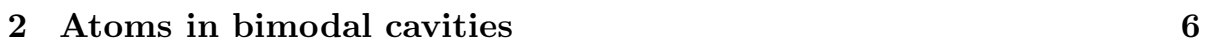

2.1 Introduction $\ldots \ldots \ldots \ldots \ldots \ldots \ldots$

$2.2 \quad$ A brief review of the JCM $\ldots \ldots \ldots \ldots \ldots$

2.3 Two-mode Jaynes Cummings models . . . . . . . . . . . . . . . 8

2.4 Two-photon micromaser $\ldots \ldots \ldots \ldots$

2.5 One-photon processes $\quad \ldots \ldots \ldots \ldots \ldots \ldots$

2.5.1 Three-level atom interacting with a bimodal cavity . . . . $\quad 9$

2.5 .2 Degenerate one-photon two-mode model . . . . . . . . 11

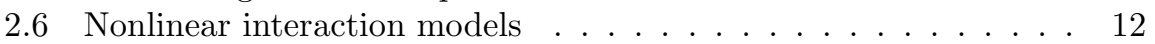

$2.6 .1 \quad$ Raman coupled model . . . . . . . . . . . . . . . . 12

2.6 .2 Nondegenerate two-mode two-photon JC model . . . . . 13

2.6.3 Intensity dependent two-mode two-photon JC model . . . 15

2.6.4 Degenerate two-mode two-photon JC model. Parity Ef-

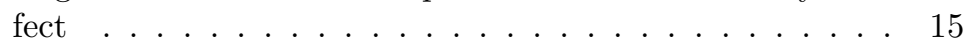

2.7 Generation of nonclassical field states in a bimodal high- $Q$ mi-

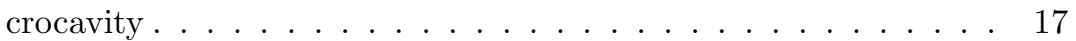

2.7 .1 Preparation of bimodal field states . . . . . . . . . 18

2.7.2 Using bimodal cavities for generating single-mode non-

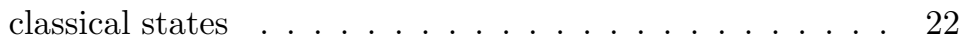

3 Ions in bidimensional traps $\quad 24$

3.1 Introduction . . . . . . . . . . . . . . . . . . . 24

3.1.1 Quantum motion of ions in Paul traps . . . . . . . . . . 25

3.1.2 Interaction of single trapped ions with external laser fields: the JC model . . . . . . . . . . . . . . . . . . 26

3.1.3 Generic bidimensional Hamiltonian models . . . . . . . . 29

3.2 Dynamics . . . . . . . . . . . . . . . . . . . . . . . . . . 30

3.2 .1 Trapped ions as quantum simulators $\ldots \ldots \ldots . . \ldots 30$

3.2 .2 Motional nonlinearities. . . . . . . . . . . . . . . . 31

3.2.3 Quantum effects in the dynamics of bidimensionally con-

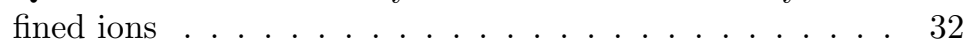

3.3 Generation of nonclassical states . . . . . . . . . . . . 34

3.3.1 Synthesis of arbitrary states of two-dimensional vibra-

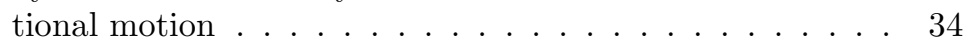

3.3.2 Bimodal generalized coherent states . . . . . . . . . . 35

$3.3 .3 \quad$ Schrödinger cat-like stated . . . . . . . . . . . . . . . . . 39

\begin{tabular}{|lll}
4 Conclusive remarks & 42
\end{tabular} 


\section{Foreword}

The history of the basic ideas of quantum theory, is studded with proposal of gedanken experiments. By this term, broadly speaking, one refers to rather idealized physical scenarios accompanied by reasonings aimed at highlighting some specific conceptual aspects abstracted from a difficult scientific problem, with disregard of any technological limitation. One of the most famous gedanken experiments was proposed by Schrödinger to draw the attention to the paradoxical results to which a blind application of quantum mechanics to the macroworld may lead. This example is known as the Schrödinger cat paradox and today it represents the archetype of all the physical situations wherein quantum superpositions of two macroscopically distinguishable states are taken into consideration. It is interesting to point out that some gedanken experiments, differently from the one involving the unlucky cat, are not only conceptual constructions but are also conceived as real, albeit impracticable, experimental schemes.

The peculiar feature of a thought experiment of this kind is the striking contrast between the simplicity of the ingredients involved (a single atom, a single harmonic oscillator, a single quantized electromagnetic mode, etc.) and the unavailability of the necessary experimental setup.

Fortunately, owing to technological progress, experiments considered unrealistic up to recently, can now be carried out. As a result, various intriguing genuinely quantum effects traceable back to the superposition principle, entanglement, quantum interference, etc., are now within experimental reach. Experiments of increasing difficultiy in cavity quantum electrodynamics (CQED) over the last years, have, for example, made it possible to test fundamental radiation-matter interaction models involving single atoms. Moreover, cavity field states possessing remarkable nonclassical features have been generated and detected.

In the domain of trapped ions, on the other hand, a single material quantum harmonic oscillator has been experimentally realized and the possibility of tailoring at will its coupling with electronic degrees of freedom has been practically demonstrated. It is therefore to be expected that these two research areas will play a decisive role in resolving the basic paradoxical aspects of quantum physics.

The scope of this paper is to review the state of the art of the theoretical investigations and of the experimental observations concerning the dynamical features of the coupling between single few-level atoms and two bosonic modes. The physical situations which we shall refer to, belong to the experimental domains of cavity quantum electrodynamics and of trapped ion physics. In the latter case we shall describe the physical properties of an ion harmonically confined in a bidimensional Paul microtrap. The motion of the center of mass of the ion is coupled to its internal dynamics using appropriate laser beam configurations. The electronic degrees of freedom of the ion are represented by pseudo-spin operators whereas the motional ones by bosonic creation and annihilation operators associated to the unperturbed bidimensional harmonic motion of the ion.

The physical scenario relative to the problems we shall be faced with in a CQED context, involves one or more two- or three-level atoms interacting, one at a time, with two quantized electromagnetic modes sustained by a high- $Q$ res- 
onator. In this case too, the dynamical properties of the system are investigated using hamiltonian models characterized by the presence of bosonic variables describing the two quantized electromagnetic modes and of pseudo-spin atomic operators. Thus the two different research areas share, at least, the mathematical language and possibly, in addition, the potentiality for realizing, with the help of different experimental setups, classes of simple but basic pseudo spin-boson hamiltonian models.

It turns out that this is indeed true, even if, as we shall see, the trapped ion context exhibits a greater practical feasibility which suggests experimental investigations beyond the possibilities of a typical CQED apparatus.

What are the motivations which justify a review of these physical situations? In particular: what is the specific role of the two bosonic modes?

A first answer is that the dynamics of several hamiltonian models describing such systems is exactly treatable and, in most cases, testable in the laboratory. A second more intriguing reason is that investigating these systems is likely to shed light on basic questions of quantum mechanics. The point to be appreciated is indeed that, studying such systems, one has the opportunity to induce entanglement and to control its evolution in a tripartite physical system atom mode 1 -mode 2 with acceptable experimental efficiency.

As we shall see, this entangled condition is essential for many theoretical proposals and experimental studies, both in CQED and trapped ion context, concerning the generation of a large class of interesting entangled states of the two bosonic modes only. We emphasize, in addition, that the presence of two modes in the physical systems under scrutiny, can be successfully exploited also to produce quantum target states of one mode only. In this last application the other mode plays an auxiliary role, leading to an effective improvement of the preparation protocol.

The quantum states of the bimodal subsystem is intrinsically interesting because, for example, it allows the production of bimodal states having nonclassical signatures. Of particular relevance is also the fact that the preparation of some entangled bimodal states yields the possibility of following the degradation of quantum coherence, under almost ideal conditions.

The importance of experimental observations of this kind might be crucial to elucidate the nature of the decoherence mechanisms, unavoidably plaguing the time evolution of any mesoscopic or macroscopic physical system. Quite recently it has been possible to follow in the laboratory the degradation of mesoscopically sized Schrödinger cat like states advancing towards the exciting goal of realizing a gedanken experiment.

We wish to emphasize that also the time evolution of the tripartite system is of interest in its own, at least under the aspect of bringing to light the mechanism governing the onset and the time development of quantum mode-mode correlation effects. In this paper, for example, we shall review the so called parity effect recently discovered in the dynamics of a two-level atom quadratically coupled to two bosonic modes. This intermode correlation effect is related to the granularity of the energy of a quantum harmonic oscillator. The occurrence of some interesting consequences of the parity effect both in CQED and trapped ion contexts, shall be presented and discussed, clarifying in this way, the motivation for reviewing together phenomena belonging to these two different domains. 
This paper is organized in two large sections. The first is devoted to systematically presenting the dynamical properties of the coupling between few-level atoms and two quantized electromagnetic modes. The second explores, in the same spirit, the physics of an ion confined in a bidimensional Paul trap and subjected to appropriate laser beam configurations. 


\section{Atoms in bimodal cavities}

\section{$2.1 \quad$ Introduction}

Over the last thirty years many theoretical investigations have been addressed toward the understanding of nonlinear effects occurring in the dynamics of an atom in a high-Q cavity. The interest toward this research area was mainly spurred by the large amount of experiments revealing the appearance of subpoissonian photon statistics [1, 2n among other intringuing features of quantum radiation-matter interaction 6, 4, 5. Both theoretical and experimental activities have concentrated on trying to understand simple non trivial models of quantum optics involving a single atom, regarded as a few effective energy levels, and one or more near resonant modes of the quantized electromagnetic field. The prototype of such systems, proposed by Jaynes and Cummings in 1963 [6], describes a two-level atom resonantly interacting with a single-mode quantized field. When the Rotating Wave Approximation (RWA) is made, the model becomes exactly solvable and its dynamical features can be analytically brought to light revealing remarkable properties. A review of the Jaynes and Cummings model (JCM) has been presented by Shore and Knight in 1993 [7]. The discovery of interesting aspects of the JCM as well as the developments in CQED experiments involving single Rydberg atoms within single-mode cavities [8], have stimulated an intense research devoted at highlighting and generalizing the original idea and physical scenario presented by Jaynes and Cummings. In particular their work has been extended to include more elaborate interactions, such as multiphoton [9, 10, 11 or intensity-dependent coupling [12, 13, 14, 15, damping and dissipation [16, 17], multiple atoms [18, 19, 20], multilevel atoms [21, 22] and multimode field [23, 24]. It is interesting to underline that historically many extensions of the JCM have been reported as examples of exactly treatable nonlinear matter-field interaction models and that some of them, including the photon loss, provide realistic description of the experiments.

After the experimental work of Brune et al [25], which have successfully realized a two-photon single mode maser operated in a high-Q cavity, a large amount of experimental and theoretical research has been done on the atomic two-photon transition processes in a cavity. The experiment successfully performed at the ENS laboratory, represents an important milestone in quantum optics because it has practically realized a two-photon cascade micromaser. Under the stimulus of the success of the experiment of Brune et al., other schemes for a two-mode two-photon maser have been presented [26].

More recently, much attention has been devoted to the generation of entangled states of two modes of two spatially separated cavities [27]. Apart from their intrinsic theoretical interest, these states play an important role in the framework of the new field of quantum communication. For example, a method for generating a maximally entangled state of two cavities has been reported as an intermediate step in the teleportation procedure proposed by Davidovich et al 28]. Recently, Zubairy et al 29] have shown the possibility of teleporting a radiation field state from a cavity to another one, provided that the generation of a two-resonator entangled state for fixed number of photons inside the two cavities, is feasible. Notwithstanding the relevance of this topic, the study of protocols aimed at entangling two separate single mode cavities, goes beyond the scope of this topical review. 
Section II of this paper is devoted to the interaction between bimodal cavities and few level atoms. In particular in Section II B a brief review of the JCM is presented whereas in Section II C its generalization to a bimodal interaction is discussed putting into evidence the interest stemming from the consideration of two modes instead of one. In Section II D a short discussion on two-photon micromaser is presented underlining its difference with respect to the one-photon micromaser. In Section II E interesting nonclassical dynamical effects of twomode JC models are reported both in correspondence to the nondegenerate case $\left(\omega_{1} \neq \omega_{2}\right)$ and in the degenerate one $\left(\omega_{1}=\omega_{2}\right)$ when one-photon processes are assumed. In Section II F we concentrate our attention on nonlinear interaction models for which the coupling mechanism between the atom and the bimodal cavity is based on two photon processes. In particular Section II F 1 is devoted to the so called Raman coupled model characterized by the fact that the net gain or loss of photons is zero in any process. The models adopted in Sections II F 2 -II F 4 are such that the respective basic processes involve the net gain or loss of a pair of photons having or not the same energy. Finally, in the last section (section II G) of this first part, several schemes aimed at generating nonclassical states in a bimodal high-Q microcavity are reported.

\subsection{A brief review of the JCM}

In the spirit of this topical review, we start devoting this section to a brief discussion of the JCM being it the simplest model describing the interaction between a single few-level atom and the quantized cavity field. This model has been extensively studied by many authors and numerous non-classical effects have been predicted and in some cases verified in laboratory [1] 2, 3, 田, 5, 6, 8]. The JCM consists in a single two-level atom, whose two states are denoted by $|+\rangle$ and $|-\rangle$, coupled to a quantized single mode of a cavity field having a frequency $\omega$ close to the transition frequency $\omega_{0}$ of the atom. The effective hamiltonian describing such a physical system, in the Rotating Wave Approximation, can be written in the form $(\hbar=1)$ :

$$
\hat{H}_{J C}=\omega_{0} \hat{S}_{z}+\omega \hat{a}^{\dagger} \hat{a}+\left[\lambda \hat{a}^{\dagger} \hat{S}_{-}+\text {h.c. }\right]
$$

where $\hat{a}$ and $\hat{a}^{\dagger}$ denote the annihilation and creation operator of the cavity mode whereas the atomic degrees of fredom are described by the pseudospin operators $\hat{S}_{z}$ and $\hat{S}_{ \pm}$satisfying the following relations

$$
2 \hat{S}_{z}| \pm\rangle= \pm| \pm\rangle, \quad \hat{S}_{ \pm}| \pm\rangle=0, \quad \hat{S}_{ \pm}|\mp\rangle=| \pm\rangle
$$

The coupling constant $\lambda$ depends on cavity volume, dipole strenghts and mode frequency [7, 30]. In the JCM the familiar Rabi oscillations characterizing the time evolution of the atomic inversion $S_{z}$, are affected by the initial distribution of photon numbers 31. It has indeed been demonstrated that the interference between all the possible Rabi frequencies involved in the dynamics of the system, causes a dephasing or collapse of the Rabi oscillations. Moreover, the discrete nature of the photon number distribution leads to a partially rephasing or revival of such oscillations. The existence of this revival property has been experimentally observed and, being related to the discretness of the field excitations, provides direct evidence for the truly quantum nature of radiation 间. 


\subsection{Two-mode Jaynes Cummings models}

A natural generalization of the JCM consists in considering a single atom interacting with two modes of the quantized cavity field. When such a physical situation is considered, several effective models can be constructed depending on the interaction mechanism characterizing the specific coupling. In particular, the Hamiltonian model describing the atom - two cavity modes coupling, assumes different forms depending on whether one-photon or two-photon processes are considered. In this last case the energy exchange between the atom and the bimodal cavity is mediated by virtual levels [32] not explicitely involved in the effective hamiltonian. In order to better clarify this point, consider one of the method followed by many authors for deriving effective models, namely the socalled "adiabatic elimination of virtual levels procedure" [33, 34, 35, 36, 37]. Its starting point consists in writing the full microscopic Hamiltonian in the dipole approximation and neglecting all the counter-rotating contributions in accordance with the RWA. Then the Heisenberg equations of the field and atomic operators in the interaction picture are considered. If the population in a given atomic level is relatively much smaller than the other ones, then, formally integrating the relative Heisenberg equations, this level, called virtual atomic level [32], can be adiabatically eliminated.

It is important to underline that the elimination of these virtual or intermediate states in the construction of two-photon effective models, leads to Stark shifts of atomic energy levels. As we will see, these shifts are proportional to photon numbers and act as intensity-dependent detuning.

The interest toward two-mode JCM originates from the several remarkable features characterizing their dynamics. In our opinion one of the most interesting property of the two-mode JC models is that they provide the possibility to use one mode to modulate, amplify or control the other one. As we will show later, this property turns out to be very useful in the production of nonclassical states of a single cavity mode. Another relevant aspect characterizing such kind of systems lies in their wide potential tunability since oscillation or amplification can occur at any two frequencies $\omega_{1}$ and $\omega_{2}$, with eventually $\omega_{1}=\omega_{2}$, such that $\omega_{1}+\omega_{2}$ matches the atomic transition frequency. Before concluding this section we wish to point out that in order to describe two-mode JC models, we will denote by $\hat{a}_{i}$ and $\hat{a}_{i}^{\dagger}(i=1,2)$ the annihilation and creation operators respectively of the $i$-th cavity mode. Moreover, when the atom is effectively describable as a two-level atom the pseudospin operators $\hat{S}_{z}$ and $\hat{S}_{ \pm}$, such that the condition (2) are satisfied, will be used.

\subsection{Two-photon micromaser}

Micromasers are quantum oscillators operating on a Rydberg atomic transitions, namely corresponding to high principal quantum numbers. In a micromaser an atomic beam is injected in a high- $Q$ microwave cavity. The atomic injection rate is low enough to ensure that there is just a single atom inside the cavity at one time. The experimental realization of these devices provides a very simple way to test fundamental predictions related to radiation-matter interaction. In particular, when the basic interaction process between each atom and the cavity field involves the emission or absorbtion of one photon per atomic transition (one-atom micromaser), the micromaser provides a near-ideal realization 
of the problem firstly theoretically treated by Jaynes and Cummings. These devices also allow the operation of two-photon oscillators involving a degenerate or nondegenerate two photon transitions between two atomic levels of the same parity. These two-photon micromasers present additional interesting features with respect to the one-photon micromaser, which are connected to the starting up of the system. Classically, the zero-field state is always stable so that an injected field is required in order to build up the oscillation. However, quantum fluctuations may drive the occurrence of a vacuum state instability, thus inducing the system to begin the oscillation. The two-photon micromaser indeed spontaneously starts without any triggering required. Moreover the two-photon micromaser provides a clean setup for studying the two photon processes. A two-photon micromaser was realized by Brune et al. 338 using a niobium superconducting cavity with $Q \sim 10^{8}$, resonant at $\omega \sim 68 G H z$. They in particular exploited a two-photon degenerate transition in Rubidium atoms.

When the two photons are emitted into different modes of the cavity (nondegenerate two-photon transition) important features of the two-photon process, such as for example the correlation between the two photons emitted in two different modes, can be studied.

It is important to emphasize that the mere fact that the atomic system emits or absorbs photon pairs leads to a field statistics very different from the one of the ordinary laser or maser fields and makes it a privileged candidate for the generation of squeezed light. In such systems the Rydberg atom plays a role similar to a coupler which correlates the two modes of light changing the photon statistics inside the cavity.

The main novelty of the nondegenerate two-photon micromaser (two different cavity modes of frequencies $\omega_{1}$ and $\omega_{2}$ respectively with $\omega_{1} \neq \omega_{2}$ ), with respect to the degenerate one $\left(\omega_{1}=\omega_{2}\right)$, is the strong correlation between the two different modes, which may produce a $50 \%$ squeezing in the difference of intensities at steady state 26].

\subsection{One-photon processes}

In this section we consider the coupling between few atoms and two modes of a high- $Q$ cavity field assuming that the interaction mechanism relies on singlephoton processes. In particular in the subsection II E 1 we focus on the case of an effective three-level atom and two nondegenerate modes of a single cavity whereas in subsection II E 2 we assume that the two cavity modes are degenerate and that the atom can be effectively represented as a two-level system.

\subsubsection{Three-level atom interacting with a bimodal cavity}

A natural extension of the JCM consists of a three-level atom coupled to one or two modes of a high- $Q$ cavity field. This generalization allows, for example, the investigation of physical phenomena associated with two-photon processes. A general treatment of the theory concerning such systems has been presented in [39, 40 where arbitrary detunings are considered. Denoting the three atomic levels by $|0\rangle,|1\rangle$ and $|2\rangle$ and limiting our discussion to the electronic dipole transition $|0\rangle-|1\rangle$ and $|1\rangle-|2\rangle$, there are three possible distinct atomic level configurations as shown in fig. 1. Following [41], these configurations are called $\Lambda$, $\Xi$ and $V$. In this context the transition $|0\rangle-|2\rangle$ is forbidden. 
When the interaction between the atomic system and the two cavity modes is considered, it is interesting to study the effects of intermode field correlations on the dynamics of the atomic system and viceversa. This particular aspect of the problem forms the basis of the paper of Lai et al [42]. In order to investigate on the occurrence of dynamical effects related to the presence of initial correlation in the physical system, the authors consider an $\mathrm{SU}(2)$ coherent state 43 as an example of a two-mode correlated state and a three-level atom in the $\Lambda$ configuration as atomic transition sequence. The $\mathrm{SU}(2)$ coherent state has the property that, indicating by $m$ the maximum possible number of excitations in each mode, if $n$ photons are observed in one mode then $(m-n)$ photons will be observed in the other one. This means that the SU(2) coherent state is an eigenstate of the total excitation number operator $\hat{a}_{1}^{\dagger} \hat{a}_{1}+\hat{a}_{2}^{\dagger} \hat{a}_{2}$ relative to the eigenvalue $m$. The three-level atomic system consists of two dipole allowed transitions, $|0\rangle-|1\rangle$ and $|1\rangle-|2\rangle$ respectively (see fig. [1(a)), resonant with two different modes of the quantized radiation field. The interaction Hamiltonian describing the coupled system, in the RWA, can be written down as

$$
\hat{H}_{I}^{\Lambda}=g_{1}\left(\hat{a}_{1}^{\dagger}|0\rangle\langle 1|+\text { h.c. }\right)+g_{2}\left(\hat{a}_{2}^{\dagger}|2\rangle\langle 1|+\text { h.c. }\right)
$$

where $g_{1}$ and $g_{2}$ are the coupling constants relative to the two different channels $|0\rangle-|1\rangle$ and $|2\rangle-|1\rangle$ respectively. The particular form of initial field correlation imposed by the authors to the two cavity modes, leads to generalized twophoton Rabi frequencies that are independent of the total photon number $m$, provided that the atom-field coupling constants are set equal. Thus, under this condition, the atomic population probability exhibits purely periodic behaviour. Moreover the revival times, that is the time instants at which the oscillations revive, are much shorter than the ones exhibited assuming the field in the twomode uncorrelated binomial state. This behaviour is of relevance in view of the fact that the photon statistics of the individual modes are the same in the two cases considered by the authors.

The $\Lambda$ configuration is also exploited in ref. [44]. In this paper the authors define the slowly varying complex amplitudes of the two-mode field as following:

$$
\hat{d}_{1}=\frac{1}{2^{\frac{3}{2}}} \sum_{j=1,2}\left(\hat{a}_{j} e^{i \omega_{j} t}+\hat{a}_{j}^{\dagger} e^{-i \omega_{j} t}\right) \quad \hat{d}_{2}=\frac{i}{2^{\frac{3}{2}}} \sum_{j=1,2}\left(\hat{a}_{j} e^{i \omega_{j} t}-\hat{a}_{j}^{\dagger} e^{-i \omega_{j} t}\right)
$$

where $\omega_{j}$ is the frequency of the $j$-th mode. By definition $\left[\hat{d}_{1}, \hat{d}_{2}\right]=\frac{i}{2}$ so that squeezing of the two-mode field occurs whenever one of the amplitude operators satisfies the condition

$$
<\left(\Delta \hat{d}_{j}\right)^{2}><\frac{1}{4}
$$

In particular the authors demonstrate the occurrence of two-mode squeezing starting from the two cavity modes prepared in a coherent state. Other interesting phenomena are found as, for example, the periodic recovery of squeezed states caused by the involved two-photon processes.

The interaction between a three-level atom and two different cavity modes has been shown to produce also normal as well as higher-order squeezing. In ref. [45, M.H. Mahran studies the interaction of a three-level V-shaped atom (see fig. 1(c)) with two quantized electromagnetic modes discussing the possibility of occurrence of squeezing. Following the definition proposed by Loudon and 
Knight [46], the author has calculated the two-mode squeezing when the atom is initially prepared in its ground state. The results obtained by Mahran brought to light the dependence of the two-mode squeezing on the initial mean photon number. In particular the observed amount of squeezing decreases as the mean value of photons initially present in the two modes increases. Finally the author considers the sum squeezing, as defined by Hillery [47, for an atom initially prepared in its ground state, proving that such a quantity is smaller than the amplitude-squared squeezing.

The ladder or $\Xi$ configuration, displayed in fig. 1 $1(\mathrm{~b})$ is investigated in 48 bringing to light that it can produce very good quantum nondemolition measurement performances.

Phase properties of the field in the two-mode three-level problem are investigated in 49]. In particular, using the Hermitian phase formalism of Pegg and Barnett [50], phase properties of each individual mode as well as their joint probability distribution and correlation function are studied. The authors show that phase properties of the field reflect the collapses and revivals of the atomic level occupation probabilities except for the Raman scattering in correspondence of which the collapses and revival of the atomic population can be completely decorrelated from the phase of the field.

Very recently [51] the master equation for the reduced density operator of the field in the two-mode micromaser pumped by ultracold $\Lambda$-type three-level atoms has been derived and the steady state photon probability distribution under the principle of detailed balance has been evaluated. Analogously to the definition given by Scully et al. [52], the system now described is called two-mode mazer (microwave amplification by the $z$ motion induced emission of radiation). When indeed the micromaser is pumped by laser cooled atoms, quantization of atomic external motion becomes necessary. The interesting result obtained by the authors is that the degree of anticorrelation between the cavity modes increases when the micromaser is pumped by ultracold atoms instead of fast atoms. This leads to much stronger sub-Poissonian statistics for each mode.

\subsubsection{Degenerate one-photon two-mode model}

In this section we focus our attention on the one-photon interaction between a single two-level atom and two degenerate modes of a lossless cavity described with the help of the following Hamiltonian model 53:

$$
\hat{H}_{d}=\omega \sum_{\mu=1,2} \hat{a}_{\mu}^{\dagger} \hat{a}_{\mu}+\omega_{0} \hat{S}_{z}+\sum_{\mu=1,2} g_{\mu}\left(e^{-i \phi_{\mu}} \hat{a}_{\mu}^{\dagger} \hat{S}_{-}+\text {h.c. }\right)
$$

This model conserves the total excitation number $\hat{N}=\hat{a}_{1}^{\dagger} \hat{a}_{1}+\hat{a}_{2}^{\dagger} \hat{a}_{2}+\hat{S}_{z}+\frac{1}{2}$. Moreover it possesses the purely bosonic constant of motion $C=\frac{g_{2}^{2}}{g_{1}^{2}+g_{2}^{2}} \hat{a}_{1}^{\dagger} \hat{a}_{1}+$ $\frac{g_{1}^{2}}{g_{1}^{2}+g_{2}^{2}} \hat{a}_{2}^{\dagger} \hat{a}_{2}-\frac{g_{1} g_{2}}{g_{1}^{2}+g_{2}^{2}}\left(\hat{a}_{1}^{\dagger} \hat{a}_{2} e^{i\left(\phi_{2}-\phi_{1}\right)}+\right.$ h.c. $)$. The existence of these two constants of motion leads to the possibility of unitarily transforming $\hat{H}_{d}$ into a system consisting of an one mode JC subsystem and a decoupled other new bosonic mode. Thus the dynamics of the model given by equation (6), may be exactly solved starting from an arbitrary initial condition.

When one mode, say the first, is prepared in a Fock state with $n \gg 1$ excitations and the other one is empty, the field dynamics is dominated by a set 
of $n$ incommensurate Rabi frequencies leading to the occurrence of oscillatory net exchanges of a large number of photons between the two modes. These oscillations of the field populations display amplitude decay and, over a longer time scale, exhibit revivals and collapses. This behaviour is displayed in fig. 2 where we have in particular chosen $n=20$. Analogous phenomena take place in the dynamics of the system when the initially populated mode is prepared in a coherent state 54. The reaction of the atomic dipole on the field and the granularity of the field itself, are at the physical origin of the highly nonclassical regime attained by the system under scrutiny. In [54 the role played by the atom-cavity field entanglement is brought to light and related to the occurrence of a cooperative intermode effect which is shown to be incompatible with a neoclassical approach.

\subsection{Nonlinear interaction models}

Over the last two decades the interest toward the dynamics of nonlinear models effectively describing the interaction between an atom and two quantized modes of a high- $Q$ cavity, has grown up in a significative way. With the emergence of the two-mode two-photon micromaser of Laughlin and Swain [55], it has become possible to experimentally observe certain nonlinear effects including field squeezing and emission spectra. Both theoretical and experimental activities concentrate on trying to understand simple non trivial models of quantum optics involving single effective two-level atoms and two independent modes of the radiation field, having or not the same frequency, coupled by means of two-photon processes. In this section we focus our attention on nonlinear twomode models, bringing to light the main features characterizing their relative dynamics.

\subsubsection{Raman coupled model}

Let's consider two quantized cavity modes of frequency $\omega_{1}$ and $\omega_{2}\left(<\omega_{1}\right)$ respectively and a three-level Rydberg atom whose energy diagram is given by fig. 3 . Following Gerry and Eberly [35], the level 2 can be adiabatically eliminated provided that the condition of large detuning $\Delta \gg E_{3}-E_{1}$ is satisfied.

Under this hypothesis the effective hamiltonian describing the system under scrutiny can be written down in the following form:

$$
\hat{H}_{R}=\hat{H}_{0}^{R}+\hat{H}_{I}^{R}
$$

with

$$
\hat{H}_{0}^{R}=\omega_{0} \hat{S}_{z}+\omega_{1} \hat{a}_{1}^{\dagger} \hat{a}_{1}+\omega_{2} \hat{a}_{2}^{\dagger} \hat{a}_{2} ; \quad \hat{H}_{I}^{R}=g_{R} \hat{a}_{1} \hat{a}_{2}^{\dagger} \hat{S}_{+}+\text {h.c. }
$$

The resulting model therefore consists of two-nondegenerate "ground"states connected by zero-photon processes where a photon absorbed in one mode is emitted in the other one with the atom making transitions through the virtual state $|2\rangle[35]$.

The system under scrutiny, effectively described by $\hat{H}_{R}$, may be interpreted as a cavity version of Raman scattering in which mode 1 is the pump field, mode 2 is the Stokes field and the anti-Stokes mode is eliminated by setting a cavity off-resonance condition [56]. As a direct consequence of the fact that we are 
dealing with zero photon process, the total photon number operator defined as $\hat{N}=\hat{a}_{1}^{\dagger} \hat{a}_{1}+\hat{a}_{2}^{\dagger} \hat{a}_{2}$ is a constant of motion [35].

The two-mode Raman coupled model considered in this section, has been extensively investigated showing the existence of collapses and revival phenomena [35], resonance fluorescence [57, population trapping [56] and several nonclassical properties of the electromagnetic field such as the appearing of antibunching light, violation of the Cauchy-Schwartz inequality and squeezing [35, 58]. As far as the collapse and revival phenomena of the Rabi oscillations, it has been proved that, differently from the JC model, in this case it can be periodic [35, 58]. In particular, assuming coherent states for both modes, the atomic inversion between the two nondegenerate ground states exhibits periodic revivals but with many secondary revivals as well. These last revivals become less significant for higher initial average photon numbers. The physical origin of such collapse and revival patterns was partially brought to light in [58] but it has been more studied, at least for the case of equal average photon numbers in both modes, by Cardimona et al. [37. As far as nonclassical properties of the electromagnetic field are concerned, Mahran [59, has, for example, studied the production of squeezing.

The Hamiltonian model (7)- (8) adopted in [35, 37, 56, 59] neglects the Stark shift terms arising from the adiabatic elimination of a virtual level. Relaxing this approximation implies indeed that the resulting two-level system is no longer two-photon resonant but it is detuned by an intensity-dependent amount. In other words the unperturbed atomic hamiltonian $\omega_{0} \hat{S}_{z}$ appearing in $H_{0}^{R}$, has to be substituted by

$$
\left(E_{3}+\frac{g_{2}^{2}}{\Delta} \hat{a}_{2}^{\dagger} \hat{a}_{2}\right)|3\rangle\left\langle 3\left|+\left(E_{1}+\frac{g_{1}^{2}}{\Delta} \hat{a}_{1}^{\dagger} \hat{a}_{1}\right)\right| 1\right\rangle\langle 1|
$$

where $g_{i}(i=1,2)$ is the atom $i-t h$ mode coupling constant. Buzek and Knight [60] have shown that this substitution may lead to quite different evolutions even for low-intensity fields. The effects of the Stark shift terms on the atomic inversion dynamics when a multilevel atom interacts with a two-mode quantized radiation field have been also investigated by Cardimona et al. 61. They have shown that these Stark terms are relatively unimportant for Raman scattering but could not be ignored in equal- or unequal-frequency absorption.

Very recently a class of coupled-channel cavity QED models describing twophoton resonant Raman processes in multiple- $\Lambda$ configuration has been solved analytically by an algebraic method based on the introduction of an angular momentum algebra for this multiwave mixing system [62].

\subsubsection{Nondegenerate two-mode two-photon JC model}

The system we consider in this subsection is a generalized JC model in which the atomic transitions are mediated by nondegenerate two-photon absorption or emission. As it is well known, in the two-photon processes at least an intermediate level, assumed to be coupled to the ground and excited states respectively by dipole allowed transitions, is involved. As shown in Fig. 1 we denote by $E_{g}$, $E_{i}$ and $E_{e}$ the lower, intermediate and upper atomic energy levels and, indicating by $\omega_{1}$ and $\omega_{2}$ the frequencies of the two cavity modes, suppose that the conditions $E_{i}-E_{g}=\omega_{1}-\Delta, E_{e}-E_{i}=\omega_{2}+\Delta$ are satisfied. It is possible 
to demonstrate that, if $|\Delta|$ is much larger than the one-photon Rabi frequency of the oscillations between $|i\rangle$ and $|g\rangle$ and between $|i\rangle$ and $|e\rangle$ respectively, the effective Hamiltonian model describing the system under scrutiny assumes the form:

$$
\hat{H}_{n d}=\omega_{1} \hat{a}_{1}^{\dagger} \hat{a}_{1}+\omega_{2} \hat{a}_{2}^{\dagger} \hat{a}_{2}+\left(\omega_{0}+\beta_{2} \hat{a}_{2}^{\dagger} \hat{a}_{2}-\beta_{1} \hat{a}_{1}^{\dagger} \hat{a}_{1}\right) \hat{S}_{z}+\left(\lambda \hat{a}_{1} \hat{a}_{2} \hat{S}_{+}+\text {h.c. }\right)
$$

The alkali-metal Rydberg energy diagram, for example, provides concrete examples fulfilling the conditions leading to an atom - bimodal cavity interaction Hamiltonian such as $\hat{H}_{n d}$ [25, 26]. Observe that the hamiltonian model given by equation (10), contains an intensity dependent detuning described by the two terms proportional to $\beta_{1}$ and $\beta_{2}$ respectively, whereas the constant $\lambda$ measures the effective two-photon atom-field coupling. This Hamiltonian is exactly solvable and a unitary operator accomplishing the canonical dressing of the two-level atom by the bimodal cavity field, has also been constructed [63].

The Hamiltonian model $H_{n d}$ was firstly introduced by Gou 64, 65]. In 664, neglecting the Stark shift arising from the presence of virtual levels, that is putting in equation (10) $\beta_{1} \sim \beta_{2} \sim 0$, the quantum dynamics of the atom and the temporal evolution of the field statistics were studied. In particular the author considers two types of initial state of the field, namely ordinary uncorrelated coherent states in both modes and the two-mode squeezed vacuum state where the number of photons in one of the modes is equal to the number of photons in the other one. It has been noted that the dynamics of the model is qualitatively different in correspondence of these two kinds of states. In more detail it has been shown that new features of quantum collapse and revival occur in presence of the two-mode squeezed vacuum. The revivals here appear periodically sharp. The origin of such a behaviour is strictly related to the particular class of Rabi frequencies involved in the dynamics of the respective physical systems. If, on the contrary, the field is initially prepared in a product of two coherent states, the atom exhibits the phenomena of collapse and revival as well, but no periodicity can be observed. The different behaviour of the atomic dynamics in correspondence to initial correlated and uncorrelated cavity field has also been put into evidence by Joshi and Puri [66] as well as by Gerry and Welch [67]. In both papers the Stark shift terms are neglected.

In 68] Ashraf has investigated the cavity field spectra for various initial fields of a nondegenerate two-photon JC model neglecting the two terms proportional to $\beta_{1}$ and $\beta_{2}$ respectively.

The results presented in [64, 67, 68] could be qualitatively or quantitatively affected by the presence of the Stark shift of the two effective two atomic levels. As shown by Gou [65], indeed, in most circumstances these terms cannot be ignored, even if for certain correlated two-mode field states, the elimination of Stark shifts is possible at least when one evaluates the ensemble averages relevant to the phase insensitive terms. However, for the phase sensitive averages such as the squeezing of cavity fields, the Stark shifts still cannot be ignored in any case. Also Cardimona et al [61] have proved that, differently from the Raman coupled model, for unequal-frequency absorption or emission, the Stark effect could not be negligible. 


\subsubsection{Intensity dependent two-mode two-photon JC model}

In this section we investigate on the nondegenerate two-mode two-photon JC hamiltonian model assuming that both the coupling strenght and the atom-field detuning may depend on the populations of the two modes. The hamiltonian describing such a general nonlinear model may be written down as [63]:

$$
\hat{H}_{i d}=\hat{H}_{1}+\hat{H}_{2}+\hat{H}_{3}
$$

with

$\hat{H}_{1}=\omega_{1} \hat{a}_{1}^{\dagger} \hat{a}_{1}+\omega_{2} \hat{a}_{2}^{\dagger} \hat{a}_{2} ; \quad \hat{H}_{2}=\omega_{0} G\left(\hat{a}_{1}^{\dagger} \hat{a}_{1}, \hat{a}_{2}^{\dagger} \hat{a}_{2}\right) ; \quad \hat{H}_{3}=\lambda\left[\hat{a}_{1} \hat{a}_{2} F\left(\hat{a}_{1}^{\dagger} \hat{a}_{1}, \hat{a}_{2}^{\dagger} \hat{a}_{2}\right) \hat{S}_{+}+\right.$h.c. $]$

where the RWA has been used. The two functions $G\left(\hat{a}_{1}^{\dagger} \hat{a}_{1}, \hat{a}_{2}^{\dagger} \hat{a}_{2}\right)$ and $F\left(\hat{a}_{1}^{\dagger} \hat{a}_{1}, \hat{a}_{2}^{\dagger} \hat{a}_{2}\right)$ appearing in equation (12), are introduced to take into account the possibility of intensity dependence both in the atom-field detuning and in the coupling constant. Let's observe that putting in particular $\omega_{0} G\left(\left\{\hat{a}_{i}^{\dagger} \hat{a}_{i}\right\}\right)=$ $\omega_{0}-\beta_{1} \hat{a}_{1}^{\dagger} \hat{a}_{1}+\beta_{2} \hat{a}_{2}^{\dagger} \hat{a}_{2}$ and $F\left(\left\{\hat{a}_{i}^{\dagger} \hat{a}_{i}\right\}\right)=I$ we recover the nondegenerate two-mode two-photon JC model discussed in the previous section. In [63] a symmetrybased detailed construction of the atom-cavity eigenstates as well as of the energy spectrum of the dressed atom is presented. The starting point of such a construction consists in finding canonical symmetry transformations for $\hat{H}_{i d}$ and the related constants of motion. In particular they prove that the operators $\hat{N}=\hat{a}_{1}^{\dagger} \hat{a}_{1}+\hat{a}_{2}^{\dagger} \hat{a}_{2}+2 \hat{S}_{z}+1$ and $\hat{D}=\hat{a}_{1}^{\dagger} \hat{a}_{1}-\hat{a}_{2}^{\dagger} \hat{a}_{2}$ are constants of motion indipendently from the specific form of the two intensity dependent functions $F$ and $G$. The existence of these two constants of motion turns out to be very useful in the construction of a unitary operator diagonalizing $\hat{H}_{i d}$. It is worth noting that the symmetry-based conclusions envisaged in 63 may be applied whatever the analytic dependence of the intensity-dependent terms on the populations of the two-cavity modes is. This result allows to highlight the role of the nonlinear contributions on the two-level atom dressed by the cavity field. In order to do this, in 63 the authors study the quantum dynamics of the initially excited two-level atom interacting, in the context of the general model, with a cavity field prepared in the tensorial product of a coherent state and a Fock state. Exploiting the flexibility of their results the authors compare the atomic evolution of several particular models finding a rich dynamical behaviour dominated by the presence of collapses and revivals of the Rabi oscillations. The occurrence of these collapses and revivals is a common phenomenon to all the models considered in [63] even if some other distinctive features characterize the time evolution of the atomic inversion in each individual model.

The existence of sensitivity to the population of the field mode initially prepared in a Fock state is also pointed out. This effect is of quantum nature and it is particularly noteworthy since, as showed in the subsequent subsection II G 2 it leads to proposals for generating number states in a single cavity mode starting from the vacuum.

\subsubsection{Degenerate two-mode two-photon JC model. Parity Effect}

Suppose now that the two modes of the cavity field have the same frequency $\omega$ assuming that they differ either by the polarization vectors or by the direction of propagation. The degeneration condition imposed to the two quantized 
electromagnetic modes makes the effective hamiltonian model describing the two-photon interaction between the bimodal cavity and a single few level atom, very different from that adopted in the previous section. The effective model obtained in this degenerate case is not a trivial generalization of the nondegenerate one (10). The explicit construction of the effective Hamiltonian for the degenerate quadratic atom-field interaction leads to the following model:

$$
\hat{H}_{d}=\hat{H}_{0}^{d}+\hat{H}_{R}^{d}+\hat{H}_{S}^{d}+\hat{H}_{I}^{d}
$$

with

$\hat{H}_{0}^{d}=\omega_{0} \hat{S}_{z}+\omega \sum_{\mu=1,2} \hat{a}_{\mu}^{\dagger} \hat{a}_{\mu} ; \quad \hat{H}_{R}^{d}=\left(r_{1} \hat{a}_{1} \hat{a}_{2}^{\dagger}+r_{2} \hat{a}_{1} \hat{a}_{2}^{\dagger} \hat{S}_{z}\right)+h . c . ; \quad \hat{H}_{S}^{d}=s \hat{S}_{z} \sum_{\mu=1,2} \hat{a}_{\mu}^{\dagger} \hat{a}_{\mu}$

and

$$
\hat{H}_{I}^{d}=\left(\sum_{\mu=1,2} \lambda_{\mu} \hat{a}_{\mu}^{2}+g \hat{a}_{1} \hat{a}_{2}\right) \hat{S}_{+}+\text {h.c. }
$$

This Hamiltonian model has been recently introduced by A. Napoli and A. Messina 69]. The structure of $\hat{H}_{d}$ reflects the fact that the atom-mode-mode field energy exchanges in the degenerate case may take place following all the different possible channels compatible with the conservation of the total excitation number operator $\hat{N}=\hat{a}_{1}^{\dagger} \hat{a}_{1}+\hat{a}_{2}^{\dagger} \hat{a}_{2}+2 \hat{S}_{z}+1$. It is easy,indeed, to demonstrate that the operator $\hat{N}$ commutes with $\hat{H}_{d}$ whatever the coupling constant $s, r_{1}$, $r_{2}, \lambda_{1}, \lambda_{2}$ and $g$ are. The term of $\hat{H}_{d}$ depending on the constant $g$, for example, arises from the possibility that the atom makes transitions involving a simultaneous gain or loss of one photon in both modes. The contributions related to the coupling constants $r_{1}$ and $r_{2}$ describe, on the other hand, mode-mode energy exchanges not involving the atom or changes in its state. These terms are known as Rayleigh terms. Stark terms describing a field-dependent detuning appear in $\hat{H}_{d}$ with the coupling constant $s$. Finally the possibility of two-photon exchanges between the atom and each cavity mode is described by the terms of $\hat{H}_{d}$ containing $\hat{a}_{\mu}^{2} \hat{S}_{+}$. The strength of this specific effective atom-field interaction mechanism is measured by $\lambda_{\mu}$.

In what follows we concentrate our attention on the case correspondent to $g=0$ and $\lambda_{1}=-\lambda_{2}=\lambda$, that is on all the physical systems effectively describable by means of the following Hamiltonian model:

$$
\begin{aligned}
\tilde{H}_{d} & =\omega_{0} \hat{S}_{z}+\omega \sum_{\mu=1,2} \hat{a}_{\mu}^{\dagger} \hat{a}_{\mu}+s \hat{S}_{z} \sum_{\mu=1,2} \hat{a}_{\mu}^{\dagger} \hat{a}_{\mu}+\left[\left(r_{1} \hat{a}_{1} \hat{a}_{2}^{\dagger}+r_{2} \hat{a}_{1} \hat{a}_{2}^{\dagger} \hat{S}_{z}\right)+h(\mathrm{~d} . \phi)\right. \\
& +\left[\lambda\left(\hat{a}_{1}^{2}-\hat{a}_{2}^{2}\right) \hat{a} S_{+}+\text {h.c. }\right]
\end{aligned}
$$

In 69] it has been shown that specific physical situations in correspondence of which the conditions $g=0$ and $\lambda_{1}=-\lambda_{2}=\lambda$ are satisfied, may be envisaged.

It is possible to convince oneself that in this case the Hamiltonian $\tilde{H}_{d}$ possesses another hermitian constant of motion, namely $\hat{C}=\hat{a}_{1}^{\dagger} \hat{a}_{2}+\hat{a}_{1} \hat{a}_{2}^{\dagger}$. The existence of the two constants of motion, $\hat{N}$ and $\hat{C}$, is at the basis of the possibility of exactly evaluating the dynamics of the system bringing to light the existence of an intrinsically quantum phenomenon, christened in 69] "parity effect", strictly connected with the granularity of the radiation field. In order to describe this nonclassical effect, let's suppose to inject a two-level atom, initially 
prepared in its ground state, into the cavity where one mode is excited in a state exactly containing $n$ photons whereas the other one is left in its vacuum state. After an appropriate $n$-dependent interval of time, the system described by $\tilde{H}_{d}$ exhibits a peculiar sensitivity to the parity of the initial total number of photons $n$. Stated another way, the physical system distinguishes between the two different initial conditions $n$ odd or $n$ even, showing macroscopically different quantum behaviours. This sensitivity to the parity of the initial photon number clearly manifests itself in the dynamics of the mean value of the population operators $\hat{a}_{\mu}^{\dagger} \hat{a}_{\mu}$. In Fig. 5 we in particular display the time evolution of the $\mu=1$ mode average photon number, correspondent to the initial conditions $n=20$ (Fig. $5(\mathrm{a})$ ) and $n=21$ (Fig. 5 (b)) respectively, putting $r_{1}=r_{2}=s=0$. These plots show that the initial behaviour of the temporal variation of the photon number in the mode $\mu=1$ is characterized by a rapid reduction to a half and it is followed by a relatively longer interval of time dominated by a negligible photon exchange between the two modes. After such a "lethargy period", the time evolution of the mode population appears to be very sensitive to the parity of the initial photon number. Indeed, choosing the initial condition $n=20$ the field mode completes the transfer of its population to the other mode provoking an inversion in the dynamical role between the two modes. On the other hand, the plot of the mean value $\bar{n}_{1}(t)$ of the operator $\hat{n}_{1}=\hat{a}_{1}^{\dagger} \hat{a}_{1}$, relative to the initial condition $n=21$, shows that, after the "lethargy period", the field mode reabsorbs the photons already transferred to the initially empty mode.

The existence of this interesting nonclassical effect, that is the sensitivity to the parity of the initial number of photons present in the excited field mode, is a peculiar feature of the atom-degenerate bimodal cavity field interaction described by $\tilde{H}_{d}$ and, as demonstrated in [69], it does not appear related to the simplifying assumption $r_{1}=r_{2}=s=0$.

We wish to underline that, as we will show in the next section, the parity effect can be successfully exploited for generating highly nonclassical bimodal field states having the form of Schrödinger cat states [70, 71].

Before concluding we wish to anticipate that in the third section of this paper, the parity effect shall appear also in the dynamics of some hamiltonian models of interest in trapped ion context. Such an occurrence is by no means surprising in view of the fact that, at a formal level, CQED and trapped ion domains make use of similar models. The important point to stress is however that, due to the different meaning attributed to the same hamiltonian model

within the two experimental realms, very different dynamical consequences of the parity effect in these two context, shall be brought to light.

\subsection{Generation of nonclassical field states in a bimodal high- $Q$ microcavity}

Investigating the dynamical behaviour of a physical system from a theoretical point of view, very often involves more or less tacit assumptions about the possibility of preparing the system in a given state at $t=0$. In this sense the experimental realization of assigned initial conditions for a system is a prerequisite for studying fundamental aspects of quantum theory 3, 72]. Besides this inherent theoretical interest, the state preparation plays a central role also in the context of the new field of Quantum Information theory encompassing Quantum 
Computing and Quantum Cryptography 73, 74, 75, 76].

So far two approaches have been mainly exploited in order to produce desired prefixed states. The first one consists in finding the appropriate Hamiltonian which can transform a given initial state into a desired one via unitary evolution. The other commonly used approach consists in making a quantum measurement on one part of an entangled system provoking the wave function collapse of the other subsystem.

Over the past few years, also under the stimulus of this rapid development in the experimental techniques, several ingenious procedures for generating nonclassical states of the electromagnetic field have been reported in the realm of cavity quantum electrodynamics $11,77,78,79,80,81,82,83,84,85,86,87,887$. Particular attention has been devoted to the creation of single-mode number states in view of the fact that they are considered as prototype of nonclassical state $777,78,80,82,83,85,86$. . These states, corresponding to an exact number of photons, possess in addition maximum information capacity and therefore they might be used for encoding and processing quantum information.

A lot of attention has moreover been devoted to the possibility of preparing superpositions of two or more macroscopically distinguishable states [70, 71, 78, 84. 89. Such states, called Schrodinger cat like states, provide an useful starting point to progress with the understanding of decoherence phenomena and to gain more insight about the elusive border between classical and quantum worlds [90].

Most of the proposals present in literature deal with the generation of nonclassical states of a single cavity mode 11, 77, 78, 80, 81, 83. The interesting dynamical properties manifested by the two-mode models discussed in the previous sections, suggest that also the generation of two-mode field states might play an important role in highlighting fundamental aspects of quantum theory. In the present section we will show that the interaction between single atoms and two independent modes of a bimodal cavity can be successfully exploited to generate several nonclassical states of the bimodal field. At the same time, resuming an idea already present in the paper of Gou [64], we will demonstrate that the bimodal cavities can be also used for producing, with reasonable simple protocols, nonclassical states of a single mode of the cavity. In particular, in subsection II G 2 we will prove that when the attention is focused on a single radiation mode, the presence of the other one plays the relevant role of a "control subsystem".

\subsubsection{Preparation of bimodal field states}

The basic idea of most of the experimental procedures aimed at generating assigned nonclassical states of one or more modes of a single cavity field, can be traced back to the possibility of manipulating the statistical properties of the radiation field by injecting, one at time, a flux of Rydberg atoms into the cavity.

In the framework of micromaser-based preparation of cavity field states, one of the methods generally used for guiding the field toward a prefixed state is the so-called conditional measurement approach which consists in selecting only those sequences wherein each atom exits the cavity in a given state and discarding all others $78,79,80$. Although most of the experimental schemes exploiting this idea are conceptually very attractive, nevertheless they are often 
not mature enough to be qualified as realistic projects. Generally speaking, indeed, the high degree of precision assumed for the experimental apparatus as well as the fact that, even in such idealized conditions, the probability of success of the experiment is often very low, seriously counter the possibility of practically implementing these proposals.

\section{a) Correlated bimodal states from atomic coherences}

Consider, for example, the controllable generation procedure aimed at producing a class of arbitrary two-mode field states in a cavity, recently proposed by Deb et al [91]. Their proposal is based on the possibility of transferring atomic coherences to the cavity field exploiting the Raman coupled model. The authors show that, injecting into the cavity $N$ three-level atoms each one initially prepared in a suitable superposition of its two nondegenerate ground states and measuring their internal state after the interaction with the cavity field, it is in principle possible to guide the field toward a state $|\phi\rangle=\sum_{n=0}^{N} \pi_{n}^{N}|n, N-n\rangle$ starting from $|\psi(0)\rangle=|0, N\rangle$. Of course $|\phi\rangle$ is normalized and its probability amplitudes $\pi_{n}^{N}$ is determined by the coupling mechanism and by details of the protocol.

The idea firstly exploited by Vogel et al. 92 of transferring atomic coherences to the field, has also been used by Zheng and Guo [93 who propose a rather formal way of preparing multimode field states having equal photon numbers in these modes.

The nonresonant interaction of a two-level atom and a bimodal cavity field is exploited by Guo and Zheng [94] to propose a method for generating, at least in principle, any linear superposition of product of coherent states of the bimodal field. The basic idea of their scheme is that the photon statistical distribution of the field will not be changed by the interaction if the detuning between the atomic transition frequency and the frequency of each mode is much larger than the coupling constant. On the contrary the phase of the field evolves with the time. The authors claim that, by state-selective measurements on the atom, it is possible to produce entangled coherent states of the bimodal field provided that both the two modes are initially prepared in a coherent state.

b) Pair Fock states

In 82 the theory underlying a new experimental conditional measurement scheme for the preparation of selected equal-intensity bimodal Fock states is presented, carefully discussing its practical feasibility. The existence of a reliable experimental method to produce pair-Fock states of controllable intensity, besides its inherent theoretical interest, is also of relevance from an applicative point of view. In the field of quantum communications, in fact, it has been very recently analyzed the possibility of transmitting, with relatively high capacity, information by multiple parallel "number state single channels" [95]. The experimental project presented in 82 is based on the nondegenerate two-mode two-photon JC coupling, described by equation (10), between single Rydberg atoms and a bimodal cavity. A peculiar and noteworthy characteristic of this specific atom-two mode field interaction mechanism is the possibility of obtain- 
ing, starting from an empty cavity, pair-Fock states of the bimodal field with a relatively high intensity. The building up of an equal-intensity pair-Fock state of the microresonator field, in accordance with the proposal under scrutiny, results from the success of a multiple iterative event. Suppose the cavity prepared in its vacuum state $|0,0\rangle$. A single excited atom enters the cavity where it simultaneously exchanges one photon with each mode as effectively described by equation (10). Immediately after the atom has left the cavity its internal state is measured using an appropriate detection technique. It is possible to convince oneself that an atomic ground state outcome disentangles the atom from the field which, in turn, collapses in the state $|1,1\rangle$. At this point a second atom is injected into the cavity with an appropriate velocity. If even the second atom is detected in its ground state exiting the cavity, then the field in the microresonator is projected onto its Fock state $|2,2\rangle$. It is not difficult to convince oneself that this procedure may be iterated so that a successful sequence of $N$ atoms detected in their ground states immediately after they have left the cavity, builds up the equal-intensity pair-Fock state $|N, N\rangle$ of the bimodal field. The authors present a detailed quantitative analysis of the scheme showing, indeed, that its probability of success is of practical interest also in correspondence to high intensity pair Fock states $(N \sim 20)$. On the other hand, notwithstanding the lowering of such a probability due to the presence of important technological limits of the experimental apparatus, the scheme maintains a reasonable compatibility with the presence of sources of imprecision in the concrete realization of the experiment.

A common aspect to many micromaser-based proposals for generating nonclassical states of the cavity field is that they require the availability of ideal devices with which the interaction time between each atom and the field may be sharply selected. In connection with the generation of bimodal Fock states, the presence of unavoidable fluctuations in the atom-cavity interaction times is taken into account from the beginning in [85]. The theory developed in this paper clearly shows that the impossibility of sharply controlling the interaction time between each atom and the bimodal cavity, as well as the presence of other important sources of experimental imprecision, reduce the probability of success for the preparation of equal-intensity pair Fock states to values that, however, are still of experimental interest. As stressed in [85], this circumstance is strictly related to the interaction mechanism chosen for manipulating the cavity field.

c) Maximally entangled states

The nondegenerate two-mode two-photon JC coupling given by equation (10) appears to be a good basis for generating other classes of purely quantum states as, for example, maximally entangled two-mode states having the form $\left|\phi_{ \pm}\right\rangle=\frac{1}{\sqrt{2}}\left[|0,0\rangle \pm e^{i \phi}|1,1\rangle\right][96]$. An attractive and relevant aspect of the scheme reported in [96], is that the phase factor $\phi$ can be manipulated from $-\pi$ to $\pi$ by simply varying an experimentally easily controllable parameter.

Very recently, the possibility of entangling two independent cavity modes has been experimentally demonstrated [88]. In particular, in 88 the authors show that, using a single circular Rydberg atom, two quantized modes of a superconducting cavity can be prepared in the following maximally entangled 
state characterized by the fact that the two modes share a single photon:

$$
\frac{1}{\sqrt{2}}\left(e^{i \phi}|0,1\rangle+|1,0\rangle\right)
$$

This EPR pair state is then revealed by a second atom probing, after a delay, the correlations between the two cavity modes.

d) Photon number statistics invariant manipulation

Exploiting the same interaction mechanism envisaged in the previous section, it is also possible to modify only the phases of the initial probability amplitudes of finding a well-defined population in the bimodal field simply injecting a single two-level Rydberg atom into the cavity [97]. As shown in [97], this kind of radiation field manipulation can be useful, for example, in the construction of states orthogonal or quasi-orthogonal to the initial one or for controlling the squeezing parameter of a given single-mode state.

e) Schrödinger cat states from parity effect

The existence of the intrinsically nonclassical "parity effect" discussed in section II F 4, is at basis of the possibility of generating entangled bimodal states having the nature of Schrödinger cats [70, 71]. We wish to underline that in this case the two modes of the bimodal cavity are degenerate and the hamiltonian model describing the two-photon interaction between the field and a single twolevel Rydberg atom is given by equation (16). Suppose to prepare the cavity leaving one mode in its vacuum state and exciting the other one in a coherent state $|\alpha\rangle$. Such an initial condition can be easily realized in laboratory. The vacuum state is, indeed, reached controlling the temperature at which the experiment is performed [4]. On the other hand, it is possible to excite one mode in a coherent state $|\alpha\rangle$, having a prefixed intensity, using classical currents [3]. The experimental protocol described in [71], starts injecting in the resonator the two-level atom prepared in its ground state. In order to elucidate in which way the parity effect previously recalled may be exploited for reaching the prefixed scope, it is convenient to cast the initial condition imposed to the system as sum of the following two contributions:

$$
|\psi(0)\rangle=e^{-\frac{|\alpha|^{2}}{2}} \sum_{n=0}^{\infty} \frac{\alpha^{2 n}}{\sqrt{(2 n) !}}|2 n, 0,-\rangle+e^{-\frac{|\alpha|^{2}}{2}} \sum_{n=0}^{\infty} \frac{\alpha^{2 n+1}}{\sqrt{(2 n+1) !}}|2 n+1,0,-\rangle
$$

In view of the linear character of quantum mechanics and as a consequence of the parity effect, the initial state evolves toward two macroscopically different states respectively stemming from the two orthogonal contributing terms appearing in equation (18). In particular, it is possible to analytically evaluate the instant of time, $t_{|\alpha|}$, at which the system exhibits in the best way such parity effect-dependent consequences. Thus, manipulating the atomic velocity in such a way that the interaction time between the cavity field and the two-level Rydberg atom exactly coincides with $t_{|\alpha|}$ and measuring the internal atomic state immediately after the atom leaves the cavity, the bimodal field is projected into 
a state $|\psi\rangle$ having the desired character of a bimodal Schrödinger cat. Indeed the state $|\psi\rangle$ can be written as a superposition of two orthogonal field states, $|\varphi\rangle$ and $|\chi\rangle$, macroscopically distinguishable in the sense that in the bimodal state $|\varphi\rangle$ the field energy is essentially concentrated in the initially empty mode whereas in the bimodal state $|\chi\rangle$ all the energy of the field may be found in the other mode.

\subsubsection{Using bimodal cavities for generating single-mode nonclassical states}

The interaction between single Rydberg atoms and bimodal high- $Q$ cavities can be also exploited with success for generating interesting nonclassical states of a single cavity mode. As we will show in this section, the presence of a second quantized mode of the electromagnetic field turns out to be very useful playing the role of an auxiliary mode whose initial condition may be appropriately chosen in order to optimize single-mode nonclassical state generation procedures.

In 83 for example, a bimodal high- $Q$ cavity is used for the controlled preparation of single-mode Fock states.

This method, developed in the context of the nondegenerate two-photon micromaser, is based on appropriate sequences of wave packet reductions so that its degree of success is accordingly measured in terms of a conditional probability. Qualitatively speaking, the main result reached in this article is the possibility of conjugating experimentally acceptable values of such a probability with a reasonable high value of the number of photons deposited in the cavity mode of interest. Following the method presented in [83] the generation, for example, of the Fock state $|10\rangle$ is greater than 0.1 . The practical reliability of the proposal reported in 83 is moreover carefully discussed from several points of view. In particular the nonideal behaviour of the atomic velocity selectors today available, has been taken into account by the authors. Their analysis puts clearly into evidence that the procedure is characterized by an experimentally significant stability against the unavoidable uncertainty in the atom-field interaction times.

In the context of this topical review, it is important to point out that, as shown in [83], the possibility of having experimentally interesting values of the probability of success, directly stems both from the peculiar two-photon twomode interaction mechanism adopted in the paper, and, above all, from the existence of an auxiliary mode. In the context of their proposal, in fact, the initial photon distribution of such a mode is singled out looking for the best compromise between purely mathematical optimization arguments, concerning the probability of success of the scheme itself, and practical limitations stemming from considerations of an experimental nature. Guided by these considerations, the bimodal cavity is prepared leaving the mode of interest in its vacuum state and exciting the auxiliary one in a coherent state $|\alpha\rangle$ with intensity $|\alpha|^{2}$ appropriately chosen. Starting from this initial condition and inverting the role of the two modes, that is considering as mode of interest the one prepared in the coherent state, it is also possible to generate a class of quantum superpositions of two single-mode coherent states each one having the same intensity of the initial state [98, 99]. In more detail, exploiting the passage of one atom only through the bimodal cavity initially prepared in accordance with the request 
previously discussed, a conditional measurement of the internal atomic state, after the atom has left the resonator, projects the mode of interest in a state having the following form:

$$
|\Psi\rangle=A\left[|\beta\rangle-e^{-i \lambda \tau}\left|\beta e^{-i \lambda \tau}\right\rangle\right]
$$

$A$ being an appropriate normalization constant. In equation (19) we have indicated respectively by $\lambda$ and $\tau$ the coupling constant and the interaction time between the atom and the bimodal cavity. Moreover $\beta=\alpha e^{-i \gamma_{-}(\tau)}$ and $\gamma_{-}(\tau)=\left(\frac{\lambda}{2}-\omega_{1}\right) \tau, \omega_{1}$ being the frequency of the mode prepared in the state (19). In the next section we present a class of dynamical problems studied in the trapped ion domain. This class includes some of the problems examined in the course of this section reinterpreted in the new experimental context. In addition we shall study the dynamical properties of other hamiltonian models extraneous to CQED domain, describing the laser driven coupling of internal and external ionic degrees of freedom, still in terms of pseudo-spin and two bosonic dynamical variables. 


\section{Ions in bidimensional traps}

\subsection{Introduction}

Recent progress in laser cooling and trapping techniques has opened up new perspectives for research in the fields of atomic physics and quantum optics. By cooling down ions to very low temperatures, indeed, purely quantum manifestations become observable 100, 101.

A single ion confined in a Paul microtrap is theoretically equivalent to a particle, moving in a harmonic potential, whose center of mass (c.m.) motion is quantized as harmonic oscillator. By irradiating the ion with classical laser beams, its internal (electronic) and motional degrees of freedom can be coupled due to the momentum exchange between the laser field and the ion.

It has been shown 102, 103, 104, 106 that there exist suitable conditions under which the effective Hamiltonian describing such a system has a form similar to the JCM where the quantized radiation field is replaced by the quantized ionic c.m. motion. This circumstance suggested the idea of studying phenomena typical of the cavity QED context with trapped ion systems. A comparison of experiments measuring quantum Rabi oscillations in the cavity QED and trapped ion contexts 104, 105 shows that the coherence time characterizing the dynamics of single trapped ions is at least one order of magnitude bigger than that typical of the behaviour of atoms crossing high $\mathrm{Q}$ cavities. An important aspect which makes the trapped ion systems so attractive and interesting is, indeed, the fact that they are very well isolated from external environment being thus very good candidates for experiments relying on the persistence of quantum coherences. Moreover, by changing the lasers configuration and parameters, a huge class of effective Hamiltonians may be engineered. These facts, together with the possibility of measuring the internal ionic state with almost unit efficiency, gave rise to many proposals extending Feynman's ideas on the use of a given quantum system to simulate the behaviour of another quantum system [107]. As far the topic of this paper is concerned, many bimodal interactions and phenomena typical of both cavity QED and nonlinear optics, can be easily obtained in the trapped ion context. For example the vibrational analogue of parametric amplification, Kerr-type nonlinearities, multimode mixing and multiphoton down conversion can be realized [108, 109, 110. Moreover a Mach-Zender interferometer acting on two modes of oscillation of the trapped ion may be simulated allowing the realization of entangled states of the ionic vibrational 2D motion [111, 112, 113. Also maximally entangled states and Bell states may be engineered by means of several different kinds of easily realizable bimodal interactions 112,113 .

It is worth noting, however, that when the spatial wave function representing the c.m. motion is no longer small compared with the driving laser wavelength, there appear nonlinear effects, characteristic of the trapped ion system, which have no counterpart in standard nonlinear optics [114, 115]. Under these conditions interesting phenomena appear due to the overlap of the atomic center of mass wave function with the laser waves causing, nonlinear modification of the coupling strength [116, 108, 109.

Summing up, trapped ion systems, on one hand allow to verify experimentally many interesting phenomena which are difficult to observe in other systems, and on the other hand they present a very rich quantum dynamics showing non- 
classical effects having no analogue in optical systems. The considerations we have done justify the growing interest toward such systems. In what follows we will present a review of the studies on bidimensionally trapped ions.

We first describe briefly the basic principles of the Paul microtrap and of the techniques for detecting the ionic internal states. The effective Hamiltonian describing the ionic dynamics is then derived and some proposals for engineering generic bimodal interactions are briefly reviewed. In subsection III B we concentrate our attention on the dynamics of the laser driven system and, in particular, on the appearance of some nonclassical effects in the ionic dynamics. Finally, in subsection III C several methods for generating different types of nonclassical states of the ion are discussed.

\subsubsection{Quantum motion of ions in Paul traps}

Due to their net charge, atomic ions can be confined by particular arrangements of electromagnetic fields. To study ions at low energy, two types of traps are usually used: the Penning trap, which uses a combination of static electric and magnetic fields, and the Paul or rf trap, which confines ions primarily through ponderomotive forces generated by inhomogeneous oscillating fields. The operation of these traps is discussed in various reviews [101, 117] and in a recent book by Ghosh 118.

Over the last few years many experimental results for ions confined in Paul microtraps have been presented. For this reason we choose to concentrate our review to physical situations in which the trapping apparatus is a Paul microtrap. In Fig. 6 we show a schematic diagram of such a trap. If a constant voltage $U>0$ and an rf alternating voltage $V \cos \left(\omega_{r f} t\right)$ are applied to the trap electrodes, an ion of charge $q$ and mass $m$ moving inside the trap sees a time dependent potential of the form

$$
\hat{\Phi}(\hat{x}, \hat{y}, \hat{z}, t)=q\left(Q_{x x}(t) \hat{x}^{2}+Q_{y y}(t) \hat{y}^{2}+Q_{z z}(t) \hat{z}^{2}\right)
$$

where $x, y$ and $z$ are the coordinates of the center of mass motion of the ion and $Q_{i i}(t)(i=x, y, z)$ are the diagonal elements of the traceless tensor of the quadrupole moment, given as

$$
Q_{i i}(t)=Q_{i i}\left[U+V \cos \left(\omega_{r f} t\right)\right] \quad(i=x, y, z)
$$

From equation (20) and (21) the equations of motion of the ionic c.m. coordinates can be derived in the form of Mathieu differential equations. Stable solutions of these equations are superpositions of a c.m. oscillation at the driving frequency $\omega_{r f}$, usually called micromotion, and oscillations of typically much smaller secular frequencies $\nu_{i}$ in each direction $i=x, y, z$. Due to the different time scales of secular and micro motion, the latter can be neglected by time averaging the equations of motion over a period of the r.f. drive. Under these conditions, the effective time averaged potential assumes the form:

$$
\hat{\Phi}(\hat{x}, \hat{y}, \hat{z})=\frac{m}{2}\left(\nu_{x}^{2} \hat{x}^{2}+\nu_{y}^{2} \hat{y}^{2}+\nu_{z}^{2} \hat{z}^{2}\right)
$$

where $\nu_{x}, \nu_{y}$ and $\nu_{z}$ are the secular frequencies in the $x, y$ and $z$ axis of the trap and, for quadrupole traps as the Paul trap, satisfy the relation $\nu_{x}+\nu_{y}=\nu_{z}$. 
Thus the quantized c.m. motion of a single trapped ion in such an oscillating electric quadrupole field can be effectively described by a 3D harmonic oscillator with the Hamiltonian

$$
\hat{H}_{c m}=\sum_{i=x, y, z} \hbar \nu_{i}\left(\hat{a}_{i}^{\dagger} \hat{a}_{i}+\frac{1}{2}\right)
$$

Here $\hat{a}_{x(y, z)}^{\dagger}$ and $\hat{a}_{x(y, z)}$ are the creation and annihilation operators of vibrational quanta in the mode corresponding to the motion along the $x(y, z)$ direction, respectively. As usual the relation between $\hat{a}_{x(y, z)}^{\dagger}$ and $\hat{a}_{x(y, z)}$ with the position operator $\hat{x}$ and its conjugate momentum $\hat{p}_{x}$ is expressible by

$$
\hat{x}=\sqrt{\frac{\hbar}{2 m \nu_{x}}}\left(\hat{a}_{x}^{\dagger}+\hat{a}_{x}\right) \quad \hat{p}_{x}=i \sqrt{\frac{m \hbar \nu_{x}}{2}}\left(\hat{a}_{x}^{\dagger}-\hat{a}_{x}\right)
$$

Analogous relations hold for the other two directions $y$ and $z$.

\subsubsection{Interaction of single trapped ions with external laser fields: the JC model}

We first consider the situation where an oscillating classical electromagnetic field propagating along the $x$ direction irradiates a single trapped ion which is constrained to move in this direction in a harmonic well with frequency $\nu_{x}$. In more detail we consider a uniform linearly polarized e.m. wave written as

$$
\hat{\mathbf{E}}(x, t)=\mathbf{E}_{0} e^{i\left(k \hat{x}-\omega_{L} t\right)}+\text { h.c. },
$$

resonantly driving a single electronic transition between two ionic internal states $|-\rangle$ and $|+\rangle$. In passing we note that the electric field is an operator acting on the Hilbert space of the harmonic oscillator as a consequence of the presence of the position operator $\hat{x}$ in the exponent. The Hamiltonian describing the system under scrutiny is the following

$$
\hat{H}=\hat{H}_{0}+\hat{H}_{i n t}
$$

with

$$
\hat{H}_{0}=\hbar \nu_{x}\left(\hat{a}_{x}^{\dagger} \hat{a}_{x}+\frac{1}{2}\right)+\hbar \omega_{0} \hat{\sigma}_{z}
$$

and

$$
\hat{H}_{i n t}=-\hat{\mathbf{d}} \cdot \hat{\mathbf{E}}(\hat{x}, t)
$$

where $\hat{\mathbf{d}}=\mathbf{d} \hat{\sigma}_{+}+$h.c., $\mathbf{d}$ being the complex matrix element of the appropriate dipole operator between the states $|+\rangle$ and $|-\rangle$.

In the Rotating Wave Approximation (RWA) the interaction Hamiltonian assumes the form

$$
\hat{H}_{i n t}=\hbar \Omega e^{i\left(k \hat{x}-\omega_{L} t\right)} \hat{\sigma}_{+}+h . a .
$$

where the coupling constant $\Omega$ is defined as

$$
\Omega=-\frac{\mathbf{d} \cdot \mathbf{E}_{0}}{\hbar}
$$


Passing to the interaction picture with respect to $\hat{H}_{0}$ we get

$$
\hat{H}_{i n t}^{I}=\hbar \Omega\left(e^{i \nu_{x} \hat{a}_{x}^{\dagger} \hat{a}_{x} t / \hbar} e^{i k \hat{x}} e^{-i \nu_{x} \hat{a}_{x}^{\dagger} \hat{a}_{x} t / \hbar}\right) e^{-i\left(\omega_{L}-\omega_{0}\right) t} \hat{\sigma}_{+}+h . a .
$$

It is useful to expand the exponential operator $e^{i k \hat{x}}$ in its normally ordered form:

$$
e^{i k \hat{x}} \equiv e^{i \eta\left(\hat{a}_{x}+\hat{a}_{x}^{\dagger}\right)}=e^{-\eta^{2} / 2} \sum_{m, l=0}^{\infty} \frac{(i \eta)^{m+l}}{m ! l !} \hat{a}_{x}^{\dagger} m \hat{a}_{x}^{l}
$$

In equation (32) we have introduced the Lamb-Dicke parameter $\eta=\sqrt{\frac{\hbar k^{2}}{2 m \nu_{x}}}$. This parameter is proportional to the ratio of the center of mass uncertainty $\Delta x=\sqrt{\hbar / 2 m \nu_{x}}$ in its vibrational ground state and the laser wavelength $\lambda=$ $2 \pi / k$. The physical situation in which

$$
\eta \sqrt{\left\langle n_{x}\right\rangle+1} \ll 1,
$$

where $\left\langle n_{x}\right\rangle$ is the mean number of oscillatory quanta along the $x$ direction, is commonly referred to as the Lamb-Dicke limit. In such a condition the ion couples with the laser behaving as a point-like object. Alternatively, $\eta$ can be seen as the ratio between the photon momentum $\hbar k$ and the ionic ground state momentum uncertainty $\Delta p_{x}=\sqrt{\hbar m \nu_{x} / 2}$. Thus, in the Lamb-Dicke regime, the effect on the center of mass wavepacket due to momentum transfer during absorption or emission of a photon is negligible. We wish to underline that very often in literature the condition $\eta \ll 1$ is referred as Lamb-Dicke limit. This condition is less restrictive than the one expressed by inequality (33). In the following we will also refer to $\eta \ll 1$ as the Lamb-Dicke condition but having in mind that the mean number of oscillatory quanta have to be small enough to satisfy (33).

Inserting equation (32) into equation(31) yields

$$
\hat{H}_{i n t}^{I}(t)=\hbar \Omega \hat{\sigma}_{+} e^{-\eta^{2} / 2} \sum_{m, l=0}^{\infty} \frac{(i \eta)^{m+l}}{m ! l !} \hat{a}_{x}^{\dagger} m \hat{a}_{x}^{l} e^{i\left[\omega_{0}-\omega_{L}+(m-l) \nu_{x}\right] t}+h . c .
$$

This Hamiltonian consists of a sum of terms oscillating at frequencies differing from $\omega_{0}-\omega_{L}$ by multiples of the vibrational frequency $\nu_{x}$. In the so called resolved-sideband regime defined by the condition $\nu_{x} \gg \Omega, \Gamma, \Gamma$ being the total linewidth of the electronic transition, the different vibrational sidebands are clearly resolved and it is possible to tune the laser in resonance with a specific vibrational sideband. Moreover, in such a physical situation, if the laser is tuned to the $k$ th sideband, with $k>0$ and $k<0$ corresponding to red and blue detuning respectively,

$$
\omega_{L}=\omega_{0}-k \nu_{x},
$$

we may neglect in equation (34) all terms oscillating with multiple vibrational frequencies. As a consequence the effective interaction hamiltonian in the interaction picture looses its time dependence, becoming

$$
\begin{array}{rlr}
\hat{H}_{i n t}^{I}(t) \simeq \hat{H}_{i n t}^{k} \equiv \hbar \Omega \hat{\sigma}_{+} \hat{f}_{k}\left(\hat{a}_{x}^{\dagger} \hat{a}_{x}, \eta\right)\left(i \eta \hat{a}_{x}\right)^{k}+h . c . & k>0 \\
\hat{H}_{i n t}^{I}(t) \simeq \hat{H}_{i n t}^{k} \equiv \hbar \Omega \hat{\sigma}_{+}\left(i \eta \hat{a}_{x}^{\dagger}\right)^{-k} \hat{f}_{-k}\left(\hat{a}_{x}^{\dagger} \hat{a}_{x}, \eta\right)+h . c . & k<0
\end{array}
$$


The operator valued function $\hat{f}_{k}\left(\hat{a}_{x}^{\dagger} \hat{a}_{x}, \eta\right)$, with $k>0$ and in its normal ordered form, can be written down as follows 114.

$$
\hat{f}_{k}\left(\hat{a}_{x}^{\dagger} \hat{a}_{x}, \eta\right)=e^{-\eta^{2} / 2} \sum_{l=0}^{\infty} \frac{(i \eta)^{2 l}}{(l+k) ! l !} \hat{a}_{x}^{\dagger} l \hat{a}_{x}^{l} \quad k>0
$$

The interaction Hamiltonian given by equation (36), due to the presence of the operator $\hat{f}_{k}\left(\hat{a}_{x}^{\dagger} \hat{a}_{x}, \eta\right)$, represents a nonlinear k-quantum Jaynes-Cummings model. There exist conditions under which this Hamiltonian reduces to the well known JC Hamiltonian and multiquantum JC Hamiltonian. To better understand this point consider the vibronic Rabi frequency defined as

$$
\Omega_{n, n+k}=\Omega\left\langle n\left|\hat{f}_{k}\left(\hat{a}_{x}^{\dagger} \hat{a}_{x}, \eta\right)\left(i \eta \hat{a}_{x}\right)^{k}\right| n+k\right\rangle=(i \eta)^{k} \Omega e^{-\eta^{2} / 2} \sqrt{\frac{n !}{(n+k) !}} L_{n}^{(k)}\left(\eta^{2}\right) .
$$

Equation (38) shows a nonlinear dependence on $n$ departing from that characterizing the usual $k$-quantum JC model. Moreover, due to the presence of the associated Laguerre polinomials in equation (38), there exist pairs of values of $\eta$ and $n>0$ in correspondence of which the laser-atom coupling practically vanishes. Let us consider the nonlinear $k=1$-quantum $\mathrm{JC}$ interaction. In the Lamb-Dicke regime, the operator $\hat{f}_{1}$ appearing in the Hamiltonian can be simplified as $\hat{f}_{1} \simeq 1$ and thus the vibronic Rabi frequency assumes the form $\Omega_{n, n+1} \simeq i \eta \Omega \sqrt{n+1}$ showing the typical behaviour of the standard JC model. The same considerations hold for a generic value of $k$ leading us to the following conclusions. The light mediated interaction between the quantized motion of the c.m. of an ion moving in a Paul microtrap and its electronic degrees of freedom shows a nonlinear character. This behaviour, traceable back to the specific adopted vibronic-coupling mechanism, is typical of this system and does not have analogues in cavity QED quantum models 114, 119. However, in the Lamb-Dicke regime, that is when the c.m. wavepacket is well localized in comparison with the laser wavelenght, the nonlinear multiquantum JC interactions reduce to the multiquantum JC models common in quantum optics. Nonetheless, it is worth stressing that ionic nonlinearities play, in general, an important role in the experiments and very often need to be taken into account to describe correctly the dynamics of the system 104, 112].

We wish to underline that many experiments have been performed, during the last few years, verifying the theoretical description of the 1D Hamiltonian model given by equation(36) [104. Moreover, a huge class of vibrational states such as Fock states, coherent states, squeezed states [104] and Schrödinger cat like states [120, have been experimentally realized and even the tomographic reconstruction of the Wigner function of a vibrational Fock state has been demonstrated 121. In most of the experiments the electronic states of the ion are coupled by using two photon stimulated Raman transitions through a third optical level as indicated in Fig. 7 104, 120, 112, 121. For sufficiently large detunings $\Delta$ such that the third intermediate state can be adiabatically eliminated, only two electronic levels are involved in the dynamics. In this sense stimulated Raman transitions are formally equivalent to a narrow single photon transition 104, 112. Moreover they recombine the advantages of strong optical electric field gradients (allowing manipulation of the state of motion) and 
microwave stability of the crucial difference frequency. It has been shown that [104, 112 the effective Hamiltonian model in this case has the same form of the one given by equation (36), where now the coupling constant $\Omega$ is given by $\Omega=\Omega_{1} \Omega_{2} / \Delta$, with $\Omega_{1(2)}=\frac{\mathbf{d}_{1(2)} \cdot \mathbf{E}_{0}^{(1(2))}}{\hbar}$ and $\Delta$ detuning from the third optical level, and $\eta=\sqrt{\frac{\hbar k^{2}}{2 m \nu_{x}}}$ with $\mathbf{k}=\mathbf{k}_{\mathbf{1}}-\mathbf{k}_{\mathbf{2}}$. We indicate with $\mathbf{d}_{1}$ and $\mathbf{d}_{2}, \mathbf{E}_{0}^{(1)}$ and $\mathbf{E}_{0}^{(2)}, \mathbf{k}_{\mathbf{1}}$ and $\mathbf{k}_{\mathbf{2}}$ the dipole matrix elements, laser amplitudes and wave vectors of the two lasers guiding the Raman transition respectively. As a consequence, the Lamb-Dicke parameter, depends, in this case, also on the relative angle between the two incident lasers through the modulus of $\mathbf{k}$. In this way it is possible at the same time to excite the ion motion along one direction only and to manipulate easily the L-D parameter simply by changing the relative directions of the beams irradiating the ion.

Raman type configurations can be employed also to implement vibrational transitions without affecting the electronic state of the ion as shown in Fig.8. The form of the effective interaction Hamiltonian in the interaction picture is, in this case, very similar to the one given by equations $(36)$ and $(37)$, but electronic inversion operators are absent:

$$
\begin{array}{llr}
\hat{H}_{i n t}^{k}=\hbar \Omega \hat{f}_{k}\left(\hat{a}_{x}^{\dagger} \hat{a}_{x}, \eta\right)\left(i \eta \hat{a}_{x}\right)^{k}+h . a . & (k>0) \\
\hat{H}_{i n t}^{k}=\hbar \Omega\left(i \eta \hat{a}_{x}^{\dagger}\right)^{-k} \hat{f}_{-k}\left(\hat{a}_{x}^{\dagger} \hat{a}_{x}, \eta\right)+h . a . & (k<0)
\end{array}
$$

We conclude this subsection sketching briefly a very efficient method, based on the so called quantum jumps technique, used in the experiments for measuring the population of the electronic states of the ion [122]. It is worth noting that such a quantity is the only one directly measurable for trapped ion systems. The method essentially consists in coupling the ground electronic level $|-\rangle$ to a third auxiliary level $|r\rangle$ by means of a third laser beam, as shown in Fig.9. The transition $|-\rangle \leftrightarrow|r\rangle$ is chosen to be a dipole allowed one. In this conditions, the presence of fluorescence detects the ion in the electronic ground level and its absence in the excited level. In other words, the detection of null fluorescence prepares the ion in the excited state since after the measurement the quantum system collapses in the eigenstate correspondent to the measured outcome. With this method also if a modest number of scattered photons are detected, the efficiency of the ability to discriminate between the two states approaches $100 \%$.

\subsubsection{Generic bidimensional Hamiltonian models}

In this subsection we wish to present the extension of the 1D Hamiltonian model given by equation (36) to the case wherein two independent modes of motion of the ionic c.m., in the effective harmonic potential present inside the trap, are excited. In the previous subsection we have assumed that the laser interacting with the ion propagates along the $x$-axis of the trap. When the laser wave vector lies on a plane of the trap, say the $x-y$ plane, the dynamics couples the motion on that plane only. In this context, usually referred to as $2 \mathrm{D}$ trapped ion, a derivation analogue to the one given in 108 leads to the following expression for the general effective Hamiltonian in the interaction picture

$$
\hat{H}_{i n t}^{m_{x}, m_{y}}=\hbar \Omega e^{i \Phi} \hat{g}_{m_{x}}\left(\hat{a}_{x}^{\dagger}, \hat{a}_{x}, \eta_{x}\right) \hat{g}_{m_{y}}\left(\hat{a}_{y}^{\dagger}, \hat{a}_{y}, \eta_{y}\right) \hat{\sigma}_{+}^{\epsilon}+\text { h.c. }
$$


with

$$
\begin{aligned}
& \hat{g}_{k}\left(\hat{a}_{j}^{\dagger}, \hat{a}_{j}, \eta_{j}\right)=\left(i \eta_{j} \hat{a}_{j}^{\dagger}\right)^{|k|} \hat{f}_{|k|}\left(\hat{a}_{j}^{\dagger} \hat{a}_{j}, \eta_{j}\right) \quad(k \geq 0) \\
& \hat{g}_{k}\left(\hat{a}_{j}^{\dagger}, \hat{a}_{j}, \eta_{j}\right)=\hat{f}_{|k|}\left(\hat{a}_{j}^{\dagger} \hat{a}_{j}, \eta_{j}\right)\left(i \eta_{j} \hat{a}_{j}\right)^{|k|} \quad(k<0)
\end{aligned}
$$

where we have assumed the resonance condition $\omega_{L}-\omega_{0}=m_{x} \nu_{x}+m_{y} \nu_{y}$ and we have used the property $\nu_{z}=\nu_{x}+\nu_{y}$, valid for all quadrupole trapping potentials. In equations (41) and (42) the operator function $\hat{f}_{|k|}$ is defined by equation (37). The parameter $\epsilon$ is equal to 1 when internal transitions are involved (Fig.7) and $\epsilon=0$ when the internal state is unchanged (Fig. 8). In the Lamb-Dicke regime, that is assuming $\eta_{x}, \eta_{y}<<1$, one gets

$$
\begin{aligned}
& \hat{H}_{i n t}^{m_{x}<0, m_{y}<0}=\hbar \Omega^{\prime}\left(\hat{a}_{x}\right)^{\left|m_{x}\right|}\left(\hat{a}_{y}\right)^{\left|m_{y}\right|} \hat{\sigma}_{+}^{\epsilon}+\text { h.c. } \\
& \hat{H}_{i n t}^{m_{x}>0, m_{y}<0}=\hbar \Omega^{\prime}\left(\hat{a}_{x}^{\dagger}\right)^{\left|m_{x}\right|}\left(\hat{a}_{y}\right)^{\left|m_{y}\right|} \hat{\sigma}_{+}^{\epsilon}+\text { h.c. } \\
& \hat{H}_{i n t}^{m_{x}<0, m_{y}>0}=\hbar \Omega^{\prime}\left(\hat{a}_{x}\right)^{\left|m_{x}\right|}\left(\hat{a}_{y}^{\dagger}\right)^{\left|m_{y}\right|} \hat{\sigma}_{+}^{\epsilon}+\text { h.c. } \\
& \hat{H}_{i n t}^{m_{x}>0, m_{y}>0}=\hbar \Omega^{\prime}\left(\hat{a}_{x}^{\dagger}\right)^{\left|m_{x}\right|}\left(\hat{a}_{y}^{\dagger}\right)^{\left|m_{y}\right|} \hat{\sigma}_{+}^{\epsilon}+\text { h.c. }
\end{aligned}
$$

with $\Omega^{\prime}=\Omega\left(i \eta_{x}\right)^{\left|m_{x}\right|}\left(i \eta_{y}\right)^{\left|m_{y}\right|}$. Hamiltonian models similar to the ones expressed by equations (44) and (45) are considered in 108, 123. Both in [123 and in 108 it is shown that, in the case $\epsilon=0$, an appropriate choice of the detunings of the lasers leads to effective Hamiltonians typical of the non linear optics context. We will analyze in more detail some examples in Section II.

It is important to underline that the derivation of the effective Hamiltonians $(40)$ with the methods presented in $108,123,112,124$ is valid under the assumption that the trap frequencies $\nu_{x}, \nu_{y}$ and $\nu_{z}$ are incommensurate. When this condition is violated, as for example in the case of bidimensional isotropic traps for which $\nu_{x}=\nu_{y}$, "unwanted" resonant terms may appear in the effective interaction Hamiltonian in the interaction picture. For isotropic traps, in order to avoid the appearance of such terms, it is necessary to use more complex laser configurations.

\subsection{Dynamics}

\subsubsection{Trapped ions as quantum simulators}

In this section we describe the dynamics of some 2D trapped ion models studied in literature over the last few years. As we have already pointed out, initial studies on single trapped ions put into evidence the formal analogy existing, under certain conditions, between these systems and some nonlinear optical systems. As a consequence, when these physical conditions are satisfied, the Hamiltonian models given by equations (43)-(46), can lead to simulations of various devices of practical interest. As an example, a (50/50) beamsplitter can be simulated. The action of the beamsplitter on vibrational Fock states has been studied by Gou and Knight in the case of 2D isotropically confined ions [111. The authors show that for these systems the unitary operator describing the beamsplitter coincides with that one describing rotations of the $x-y$ axes 
of the trap around the $z$ axis in the real space. Similarly in 113,112 a MachZender interferometer acting on the two modes of oscillation of a single trapped ion, has been considered. The analogy with the Mach-Zender interferometer for bosons is that the two input modes to the boson interferometer are replaced by the $x$ and $y$ modes of ion oscillation.

Another example of the use of trapped ions to simulate the dynamics of optical sistems is given in [110]. In this paper, indeed, Agarwal and Banerji demonstrate that a single bidimensionally confined ion provides a natural way of realizing the quantum limit of second harmonic generation in a degenerate optical parametric oscillator (OPO). They show that simulating the quantum regime of OPO allows to demonstrate the existence of an effect strictly quantum in nature.

\subsubsection{Motional nonlinearities}

All the examples concerning the use of trapped ions as quantum simulators rely on the assumption that the Lamb-Dicke condition is satisfied. However, when the spatial extension of the atomic wave function representing the c.m. motion is no longer small compared with the driving laser wavelenght, nonlinear effects having no counterpart in standard nonlinear optics emerge. In [108, 119] several Raman-type excitations for inducing various kinds of nonlinear interactions in the quantized motion of a trapped ion are presented. It is there shown that standard effects can be realized, including coherent displacement, Kerr nonlinearities and parametric modes couplings. In the laser-assisted motional dynamics, however, far from the Lamb-Dicke regime, additional nonlinearities appear because of the effects on the atomic wave function due to the driving laser waves. One of the consequences of these nonlinearities consists in a nonlinear partitioning of the phase space, the action of the time evolution being different in neighboring phace-space zones 108. To better understand the origin of the phase space partioning let us consider first, for semplicity, the single mode dynamics, where only the motion in the $x$ direction is affected by the lasers $\left(\eta_{y}=\eta_{z}=0\right)$. In this case the Hamiltonian is given by equation (39) with $\hat{f}_{k}\left(\hat{a}_{x}^{\dagger} \hat{a}_{x}, \eta\right)$ given by equation (37). If the Raman lasers are tuned to the first vibrational sideband, $\omega_{L}=\omega_{L 1}-\omega_{L 2}=\nu_{x}$, the structure of the time evolution operator shows some formal resemblance to a nonlinearly modified coherent displacement operator:

$$
\hat{U}_{i n t}(t)=\hat{D}\left[-\eta_{x} \Omega^{*} \hat{f}_{1}\left(\hat{n}_{x}, \eta_{x}\right) t\right]=\exp \left[-\eta_{x} \Omega^{*} t \hat{a}_{x}^{\dagger} \hat{f}_{1}\left(\hat{n}_{x}, \eta_{x}\right)+\text { h.c. }\right]
$$

Note that for small values of the Lamb-Dicke parameter, $\eta_{x} \ll 1$, the operator (48) may be replaced by the usual displacement operator $\hat{D}\left(-\eta_{x} \Omega^{*} t / 2\right)$. Insight into the nonlinear modifications of the displacement can be obtained by considering the expectation value of the operator function $\hat{f}_{1}\left(\hat{n}_{x}, \eta_{x}\right)$ in a coherent state $|\alpha\rangle$ :

$$
\left\langle\alpha\left|\hat{f}_{1}\left(\hat{n}_{x}, \eta_{x}\right)\right| \alpha\right\rangle=\left(\eta_{x}|\alpha|\right)^{-1} J_{1}\left(2 \eta_{x}|\alpha|\right) e^{-\eta_{x}^{2} / 2}
$$

This function shows the oscillatory behaviour typical of the Bessel function, where the zeros are of special importance. Indeed these zeroes lead to the partitioning of phase-space into different (radial) zones, with their boundaries being defined by the zeros. In neighbouring phase-space zones the actions of the time evolution appear to be significantly different from each other. For example, 
one can see from numerical calculations that in two adjacent zones a nonlinearly modified "displacement" operator acts in opposite directions. Consequently, a quantum state initially located on the boundary between two zones will be split in two subspaces which eventually give rise to quantum interferences.

When two or three dimensions are involved in the Raman induced dynamics, the partioning effect appears in the phase space of each motional degree of freedom. As a consequence, the coupling between different motional modes will be strongly influenced by the interplay of these nonlinear effects. So, as pointed out by Wallentowitz and Vogel in 108, besides the feasibility of realizing phenomena well known from linear optics in the motion of bidimensionally trapped ions, these systems open up novel possibilities for studying new nonlinear quantum phenomena.

\subsubsection{Quantum effects in the dynamics of bidimensionally confined ions}

In this subsection we discuss some quantum effects recently brought to light in the dynamics of 2D isotropically confined ions [126, 127]. Quantum phenomena similar to the ones we are going to describe have been also studied in cavity QED context. Nonetheless, in the context of trapped ions they acquire a new physical meaning and they appear to be much easier to verify experimentally. Before describing the system under scrutiny we want to underline that here, as well as in many of the theoretical proposals we will describe in this review paper, an optical transition coupling directly the electronic states is considered. In the experiments, however, two photon Raman transitions are preferred. However, it is always possible to substitute the direct coupling scheme with the two photon Raman scheme obtaining the same effective Hamiltonian models [104.

We consider a two-level ion confined in a bidimensional isotropic harmonic potential characterised by the trap frequency $\nu_{x}=\nu_{y}=\nu$. It is well known that the bidimensional harmonic oscillator may be associated to a generalised Schwinger angular momentum operator $\hat{\mathbf{J}} \equiv\left(J_{1}, J_{2}, J_{3}\right)$ as follows

$$
\hat{J}_{1}=\frac{\hat{a}_{x}^{\dagger} \hat{a}_{y}+\hat{a}_{y}^{\dagger} \hat{a}_{x}}{2} \quad \hat{J}_{2}=\frac{\hat{a}_{x}^{\dagger} \hat{a}_{y}-\hat{a}_{y}^{\dagger} \hat{a}_{x}}{2 i} \quad \hat{J}_{3}=\frac{\hat{a}_{x}^{\dagger} \hat{a}_{x}-\hat{a}_{y}^{\dagger} \hat{a}_{y}}{2}
$$

It is worth stressing that these generalized angular momentum operators acquire, in the context of $2 \mathrm{D}$ isotropically confined ions, a direct and interesting physical meaning. The operator $\hat{J}_{3}$ is indeed simply half the difference between the number of vibrational quanta along the $x$ and $y$ axis of the trap. The operator $\hat{J}_{2}$ is proportional to the $z$-component of the ionic adimensional angular momentum operator $\hat{L}_{z} \equiv \frac{1}{\hbar}\left(\hat{x} \hat{p}_{y}-\hat{y} \hat{p}_{x}\right) \equiv-i\left(\hat{a}_{x}^{\dagger} \hat{a}_{y}-\hat{a}_{y}^{\dagger} \hat{a}_{x}\right)=2 \hat{J}_{2}$. Finally the operator $\hat{J}_{1}$ has the property of being proportional, under certain conditions, both to the quantum covariance $C(\hat{x}, \hat{y})=\langle\hat{x} \hat{y}\rangle-\langle\hat{x}\rangle\langle\hat{y}\rangle$ between the position operators $\hat{x}, \hat{y}$ and to the quantum covariance $C\left(\hat{p}_{x}, \hat{p}_{y}\right)=\left\langle\hat{p}_{x} \hat{p}_{y}\right\rangle-\left\langle\hat{p}_{x}\right\rangle\left\langle\hat{p}_{y}\right\rangle$ between the momentum operators $\hat{p}_{x}, \hat{p}_{y}$ 126.

It has been shown that by irradiating the ion with an appropriate configuration of laser beams the physical system can be studied, in the L-D limit and in the interaction picture, by the following Hamiltonian model [144, 126, 127, $129,128,130$ :

$$
\hat{H}_{i n t}=\hbar \Omega^{\prime}\left(\hat{a}_{x} \hat{a}_{y} \hat{\sigma}_{+}+\text {h.a. }\right)
$$


with $\Omega^{\prime}=\Omega \eta^{2}$. This Hamiltonian is unitarily equivalent to the last term of equation (16) responsible for the parity effect presented in section II D 4, in the context of cavity QED. In this case, however, Stark shift terms present in equation (16) are absent and the parity dependent quantum effect discussed in section II D 4 is much "cleaner". Moreover it turns out that new physical observable, having a direct physical meaning only in the trapped ion context, show an interesting nonclassical behaviour. In particular in 126] it is shown that, starting from an initial highly excited unidimensional Fock state $\mid n_{\bar{x}}=$ $\left.N, n_{\bar{y}}=0\right\rangle$ with $N \gg 1$, along the direction $\bar{x}$ having an angle of $\pi / 4$ relative to the $x$ axis, the time evolution of the mean value of the operator $\hat{J}_{1}$ may be written as follows:

$$
\left\langle\hat{J}_{1}(t)\right\rangle \simeq \frac{N}{2} \cos ^{N-1}\left(\frac{\Omega^{\prime} t}{N}\right)
$$

From equation (52) it is easy to deduce that at the time instant $t_{N}=\frac{\pi N}{\Omega^{\prime}}$ one gets $\left\langle\hat{J}_{1}(t)\right\rangle \simeq(-1)^{N-1} \frac{N}{2}$. This result clearly evidences that the system under scrutiny possesses an inherent peculiar sensibility to the parity of the initial total number $N$ of vibrational quanta. There exists indeed a $N$-dependent instant of time $t_{N}$ at which $\left\langle\hat{J}_{1}(t)\right\rangle$ assumes values crucially related to the evenness or oddness of $N$. In more detail, $\left\langle\hat{J}_{1}\left(t_{N}\right)\right\rangle$ is equal to $-N / 2$ when $N$ is even and $+N / 2$ when $N$ is odd. Fig 10 shows the time evolution of the quantity $\left\langle\hat{J}_{1}(t)\right\rangle$. Remembering the link between $\left\langle\hat{J}_{1}(t)\right\rangle$ and the quantum covariances $C(\hat{X}, \hat{Y})$ and $C\left(\hat{P}_{x}, \hat{P}_{y}\right)$ and taking into consideration that the maximum (minimum) mean value of $\hat{J}_{1}$ is $N / 2(-N / 2)$, this result can be readily interpreted in the following way. The dynamics of the system under scrutiny is characterised by the existence of a $\mathrm{N}$-dependent instant of time at which the ionic oscillatory motions along the $x$ and $y$ axis are maximally or minimally correlated in position and momentum in dependence on the oddness or evenness of $N$ respectively.

Another interesting quantity displaying a parity dependent temporal evolution is the variance of the operator $\hat{J}_{2}$ which is proportional to the variance of the axial orbital angular momentum operator $\hat{L}_{z}$. In a system identical to the one previously described, the mean value of $\hat{J}_{2}$ is null at any time instant $t$, while at the time instant $t_{N} / 2=\pi N / 2 \Omega$ the square of the variance assumes the value 127

$$
\left(\Delta J_{2}\right)^{2}\left(t_{N} / 2\right) \simeq\left(1-(-1)^{N}\right) \frac{N^{2}}{8}+O(N)
$$

This means that $\left(\Delta J_{2}\right)^{2}\left(t_{N} / 2\right)$ is of the order of $N \gg 1$ when the initial number of vibrational quanta is even and of the order of $N^{2}$ when it's odd. In other words the variation of one vibrational quantum only in the initial conditions of the system, quite drastically modifies the dispersion around zero of the measured values of the ionic angular momentum $z$-component at a subsequent specific instant of time. In 129 we have shown that at this time instant, $t_{N} / 2$, the vibrational and electronic degrees of freedom of the ion are disentangled for odd $N$ and maximally entangled for even $N$. At $t=t_{N} / 2$, indeed, the probability of finding the ion in its ground electronic state is $1 / 2$ or 1 in correspondence to $N$ even or odd respectively. We have also checked the robustness of this parity dependent entanglement effect against the non-dissipative decoherence due to laser intensity fluctuations and imperfect initial state preparation finding that, for values of the laser parameters currently used in the experiments, the 
nonclassical effect, althought attenuated, is still present [131, 132].

\subsection{Generation of nonclassical states}

\subsubsection{Synthesis of arbitrary states of two-dimensional vibrational motion}

As we have already mentioned in section III A, trapped ions are exceptionally well suited systems to study fundamental aspects of quantum theory. By irradiating the ion with laser fields coupling its internal and external degrees of freedom, a single ion can be cooled to the vibrational ground state of the trap and its motion can be manipulated coherently to generate nonclassical states of motion. The ability to synthesize arbitrary motional states and to control their evolution not only allow to verify experimentally fundamental concepts of quantum mechanics, but also opens new horizons for potential technical applications such as quantum information processing. For these reasons, during the last few years, several proposals for engineering arbitrary nonclassical states of the 1D, 2D or 3D oscillatory motion of the ionic center of mass have been presented (see [119] and references therein for a review). In what follows we concentrate our attention on bidimensional engineering schemes.

The first scheme for preparing an arbitrary motional state of a bidimensionally trapped ion was proposed by Gardiner, Cirac and Zoller 133 as a key prerequisite for quantum measurement of an arbitrary motional observable. The method, conceptually similar to the one proposed by Law and Eberly in the context of cavity QED [134, consists in preparing a two-mode target state of the form

$$
\left|\Psi_{\text {target }}\right\rangle=\sum_{n_{x}=0}^{N_{\max }} \sum_{n_{y}=0}^{M_{\max }} c_{n_{x}, n_{y}}\left|n_{x}, n_{y}\right\rangle,
$$

starting from the initial vibrational and electronic ground state $\mid n_{x}=0, n_{y}=$ $0,-\rangle$. Such a state is generated by means of an appropriate sequence of laser pulses used to coherently distribute the initial amplitude to form a desired arbitrary superposition.

Although conceptually elegant, the method proposed in 133 is difficult to implement since the number of laser pulses necessary for preparing the bimodal target state depends exponentially on the dimensionality of the subspace of the Fock space in which the target state is embedded. Ideal synthesis of motional quantum states assumes indeed negligible dissipation effects and perfect control of areas and phases of the laser pulses and switching times. Fluctuations of these quantities cause some technical noise affecting the efficiency of the schemes. For these reasons it is desirable to obtain preparation schemes based on a number of laser pulses as small as possible. In this spirit, during the last few years, novel approaches allowing to reduce consistently the number of operations required for synthesizing desired target states have been proposed [135, 137, 138]. In particular very recently Zheng presented a method for preparing arbitrary bidimensional vibrational states of a trapped ion of the form (54) requiring only $\left(M_{\max }+2\right)\left(N_{\max }+1\right)$ laser pulses [138]. It is worth noting, however, that a state of the form (54) involves $\left(M_{\max }+1\right)\left(N_{\max }+1\right)$ complex coefficients, each controlled by the amplitude and phase of a laser pulse. Thus it should exist, in 
principle, a better approach requiring only $\left(M_{\max }+1\right)\left(N_{\max }+1\right)$ operations [138.

All the procedures we have described till now concern the generation of arbitrary bimodal oscillatory states of the c.m. motion of an ion confined in a trapping potential. They rely on the possibility of engineering suitable interaction Hamiltonians, generally involving the two mode bosonic operators and the atomic ones, to control the evolution from the initial state to the desired target state. It has been shown that, like in the case of bimodal cavity fields, such bimodal interactions can also be used to synthesize more efficiently arbitrary unidimensional vibrational states. In a recent proposal, Hladky, Drobny and Buzek show how to engineer an arbitrary unitary operator $\hat{V}$, transforming any state $|\Psi\rangle$ of a single bosonic mode, e.g. a 1D vibrational state of a trapped ion along the $x$ direction, to another state $\left|\Psi^{\prime}\right\rangle=\hat{V}|\Psi\rangle$ 140. The realization of the unitary transformation $\hat{V}$ is based on an enlargement of the Hilbert subspace of the system. In the case under scrutiny the ionic c.m. mass mode of oscillation along $x$ becomes entangled with auxiliary degrees of freedom (ancilla) which are represented by the vibrational mode along the $y$ direction and three internal electronic levels of the ion. The physical realization of the desired operator $\hat{V}$ consists again of a sequential switching of laser fields irradiating the ion, the correspondent interaction Hamiltonians being typical and quite easy to realize in the trapped ion context. The overall algorithm involves a conditional measurement of an internal ionic state, thus the process is not unitary. It is, however, universal since the probability of the conditional measurement does not depend on the initial vibrational state.

\subsubsection{Bimodal generalized coherent states}

In this subsection we will review some recent proposals for realizing, in the context of trapped ions, generalized coherent states (GCS) [141] such as pair coherent states, $\mathrm{SU}(1,1)$ coherent states and $\mathrm{SU}(2)$ coherent states. Over the last several years there has been much discussion on GCS of the quantized electromagnetic field since they exhibit strong nonclassical properties such as sub-Poissonian statistics, squeezing, violation of Cauchy-Schwarz inequality and so on [145]. Unfortunately they are not very easy to realize in practice in the optical context. We have already seen, however, that it is possible to imagine rather simple mechanisms by which a great variety of effective Hamiltonians can be engineered in the trapped ion context. Such interaction models can be used, as we shall see in the following, to generate several classes of vibrational states including generalized coherent states. There exist basically two main ways to generate a given vibrational state with trapped ions. The first way consists in the coherent evolution due to an appropriately engineered interaction Hamiltonian, starting from a given initial state. Eventually a conditional measurement can be performed to project the ionic vibrational state in the desired target state. The second way takes into account also the effects of decoherence due to spontaneous emission in the process of state generation. An advantage of this last method is that the desired states are obtained as steady state solutions of the master equation containing dissipative terms due to spontaneous emission. These target states are called dark states because their generation experimentally corresponds to the extinction of fluorescence by the ion. Whenever a given 
class of states is defined by an eigenvalue equation, it is possible to imagine, in the trapped ion context, a configuration able to realize the dark target state starting from a proper initial condition of the system. To better understand this point we remind that, as the damping of vibrational quanta can be significantly suppressed in an ion trap, the dominant decay process is the spontaneous emission from the two-level ion. Thus the time evolution of the system, in the interaction picture, can be described by a density operator $\hat{\rho}$ obeying the master equation

$$
\frac{d \hat{\rho}}{d t}=-\frac{i}{\hbar}\left[\hat{H}_{i n t}^{I}, \hat{\rho}\right]+\frac{\Gamma}{2}\left(2 \hat{\sigma}_{-} \hat{\varrho} \hat{\sigma}_{+}-\hat{\sigma}_{+} \hat{\sigma}_{-} \hat{\rho}-\hat{\rho} \hat{\sigma}_{+} \hat{\sigma}_{-}\right)
$$

where $\Gamma$ is the spontaneous decay rate of the excited ionic state and $\varrho$ is the modified density operator

$$
\hat{\varrho}=\frac{1}{4} \int_{-1}^{1} \int_{-1}^{1} d u d v W(u, v) e^{i k(u \hat{x}+v \hat{y})} \hat{\rho} e^{-i k(u \hat{x}+v \hat{y})}
$$

accounting for the momentum transfer in the $x-y$ plane due to spontaneous emission. In equation (56) $W(u, v)$ describes the angular distribution of the spontaneous emission. The steady state solution of equation (55) has the form $\hat{\rho}_{S}=|-\rangle|\Psi\rangle\langle\Psi|\langle-|$, where $\hat{\rho}_{S}$ satisfies the restriction $\left[\hat{H}_{i n t}^{I}, \hat{\rho}_{S}\right]=0$. If the interaction Hamiltonian has the form

$$
\hat{H}_{i n t}^{I}=\hbar \Omega\left[\hat{A}_{v i b}-\epsilon\right] \sigma_{+}+h . a .
$$

, where $\hat{A}_{v i b}$ is a generic vibrational operator, then the stationary state of the system will satisfy the eigenvalue equation $\hat{H}_{\text {int }}^{I}|\Psi\rangle=\epsilon|\Psi\rangle$.

\section{a) $S U(2)$ coherent states}

We have seen in subsection III B 1 that a Mach-Zender interferometer can be simulated with 2D trapped ion systems. An SU(2) interferometer is a peculiar kind of Mach-Zender interferometer of particular relevance in quantum optical theory. The vibrational SU(2) states are anticorrelated bimodal states formally identical to the photon states on the output ports of a $\mathrm{SU}(2)$ interferometer with number states inputs. As a consequence a way to generate vibrational $\mathrm{SU}(2)$ states is to prepare an initial bimodal Fock state and then to realize an effective Hamiltonian "simulating" the action of a SU(2) interferometer. In [111 it is shown that the mode-mixing process on the output ports of the interferometer is replaced, in the context of trapped ions, by a real-space rotation in the $x-y$ plane. This means, in other words, that a bimodal Fock state excited along the $x-y$ axis is an $\mathrm{SU}(2)$ state in the coordinate system rotated of an angle $\theta$ with respect to $x-y$. We wish to remind that, as seen in subsection III B 3 , an isotropic bidimensional harmonic oscillator may be associated to a generalized Schwinger angular momentum operator $\hat{J}$ whose components are defined in equation (50). The two mode Fock state $\left|n_{x}, n_{y}\right\rangle$ can be described as a pseudo angular momentum state $|j, m\rangle$ which is the common eigenstate of the angular momentum operators $\hat{J}^{2}$ and $\hat{J}_{3}$ with correspondent eigenvalues $j=\frac{1}{2}\left(n_{x}+n_{y}\right)$ 
and $m=\frac{1}{2}\left(n_{x}-n_{y}\right)$. The generalized $\mathrm{SU}(2)$ coherent states are defined by the action of the generalized $\mathrm{SU}(2)$ displacement operator as follows

$$
|\tau, j\rangle=e^{\left(\beta \hat{J}_{+}-\beta^{*} \hat{J}_{-}\right)}|j,-j\rangle
$$

where $\hat{J}_{+}=\hat{J}_{1}+i \hat{J}_{2}, \hat{J}_{-}=\hat{J}_{1}-i \hat{J}_{2}, \beta=\alpha e^{(-i \gamma)}(0 \leq \alpha \leq \pi, 0 \leq \gamma \leq 2 \pi)$ and $\tau=\tan (\alpha / 2) e^{(-i \gamma)}$. Reminding that the operator $\hat{\widehat{J}}_{2}$ is proportional to the $z$ component of the orbital angular momentum operator generating rotations in the $x-y$ plane, it is not difficult to convince oneself that the method proposed in 1111 for generating $\mathrm{SU}(2)$ states allows also to engineer "real" $\mathrm{SU}(2)$ coherent states, that is states $|\tau, j\rangle$ with $\tau$ real.

Very recently another method for generating dark $\mathrm{SU}(2)$ states of the bidimensional oscillatory motion of a single trapped ion has been proposed [143]. Such states are eigenstates of both the total number of vibrational quanta and the vibrational operator $\hat{A}_{v i b}=\hat{a}_{x} \hat{a}_{y}^{\dagger}+$ h.a.. Due to the degeneration of their eigenvalue spectrum, however, the dark states obtained as steady state solutions of the master equation (55) would be incoherent superpositions of $\mathrm{SU}(2)$ states. It is shown in 143 that, for large values of the L-D parameter, motional nonlinearities come into play removing the eigenvalues degeneration. In these circumstances it is possible to show numerically that the steady state solutions approximate well $\mathrm{SU}(2)$ states [143].

b) Dark pair and $S U(1,1)$ coherent states

Let us now discuss two similar methods for generating dark pair coherent states and $\mathrm{SU}(1,1)$ coherent states 144,145 . We firstly review the procedure for engineering pair coherent states 144 .

The pair coherent states $|\xi ; q\rangle$ are defined as the eigenstates of both the pair annihilation operator $\hat{a}_{x} \hat{a}_{y}$ and the number difference $\hat{Q}=\hat{n}_{x}-\hat{n}_{y}$ and, in the Fock state basis have the form:

$$
|\xi ; q\rangle=N_{q} \sum_{l=0}^{\infty} \frac{\xi^{l}}{\sqrt{l !(l+q) !}}|l+q, l\rangle
$$

where $N_{q}=\left[|\xi|^{-q} I_{q}(2|\xi|)\right]$ is the normalization constant ( $I_{q}$ is the modified Bessel function of the first kind of order $q$ ). It is then clear that, by engineering an Hamiltonian model of the form

$$
\hat{H}_{i n t}^{I}=\hbar \Omega\left[\hat{a}_{x} \hat{a}_{y}-\xi\right] \hat{\sigma}_{+}+h . a .
$$

the dark state obtained is an eigenstate of $\hat{a}_{x} \hat{a}_{y}$ with eigenvalue $\xi$. This means that, since the number difference operator $\hat{Q}$ is a constant of motion in processes involving simultaneous pair annihilation or creation, starting from an eigenstate of $\hat{Q}$, like $\left|\Psi_{0}\right\rangle=|q, 0\rangle$, one obtains as dark steady state the pair coherent state $|\xi, q\rangle$.

A similar technique is used in 145 to create one mode and two mode $\mathrm{SU}(1,1)$ intelligent states of the vibrational ionic motion. $\mathrm{SU}(1,1)$ intelligent states are a class of $\mathrm{SU}(1,1)$ coherent states defined by the property that an $\mathrm{SU}(1,1)$ uncertainty relation is equalized [147. It can be shown [148] that these states are solutions of the following eigenvalue problem

$$
\left(\alpha \hat{a}_{x} \hat{a}_{y}+\beta \hat{a}_{x}^{\dagger} \hat{a}_{y}^{\dagger}\right)|\Phi\rangle=\zeta|\Phi\rangle
$$


Moreover, they are eigenstates of the number difference operator $\hat{Q}$. Thus, preparing the system in an eigenstate of $\hat{Q}$ and irradiating the ion with a configuration of lasers realizing the Hamiltonian model

$$
\hat{H}_{\text {int }}^{I}=\hbar\left[\Omega \hat{a}_{x} \hat{a}_{y}+\bar{\Omega} \hat{a}_{x}^{\dagger} \hat{a}_{y}^{\dagger}-\zeta\right] \hat{\sigma}_{+}+\text {h.a. }
$$

the steady state solution, indicated by extintion of fluorescence emission by the ion, is an intelligent $\mathrm{SU}(1,1)$ state.

c) Generation of pair coherent states via QND measurements

To conclude this subsection we wish to describe a recent alternative technique for generating pair coherent states [149, 150]. The scheme is based on a Quantum Non Demolition (QND) measurement procedure and allows actually to engineer several classes of vibrational states 151. The ion is irradiated by two laser beams propagating along the $x$ and $y$ axis of the trap and realizing, in the L-D limit and in the interaction picture an interaction of the form 149, 150, 151, 152,

$$
\hat{H}_{i n t}^{I}=\left|\Omega_{L x}-\Omega_{L y}-\chi\left(\hat{n}_{x}-\hat{n}_{y}\right)\right| \hat{\sigma}_{+}+\text {h.a. }=\left|\Omega_{L x}-\Omega_{L y}-\chi \hat{Q}\right| \hat{\sigma}_{+}+\text {h.a. }
$$

where $\Omega_{L x}=d E_{x} / \hbar, \Omega_{L y}=d E_{y} / \hbar$ and we have assumed the condition $\Omega_{L x} \eta_{x}^{2}=$ $\Omega_{L y} \eta_{y}^{2} \equiv \chi$, with $\Omega_{L x} \neq \Omega_{L y}$. The nonlinear JCM of equation (63) is exactly solvable and it is not difficult to prove that, if the system is initially prepared in a state of the form $\left|n_{x}, n_{y},-\right\rangle$, then Rabi oscillations of the electronic occupation occur, the vibrational state remaining unchanged:

$$
\left|\Psi_{-}(t)\right\rangle=\cos \left[\Omega\left(n_{x}, n_{y}\right) t\right]\left|n_{x}, n_{y},-\right\rangle-i \sin \left[\Omega\left(n_{x}, n_{y}\right) t\right]\left|n_{x}, n_{y},+\right\rangle,
$$

with $\Omega\left(n_{x}, n_{y}\right)=\left|\Omega_{L x}-\Omega_{L y}-\chi q\right|$ and $q=n_{x}-n_{y}$. We have seen in section III A that, in a typical trapped ion experiment, a measurement of the internal electronic state is realized coupling the ground state with a third level by an auxiliary laser beam 122 . If a null fluorescence is detected then we may claim with certainty that the ion is not in its ground state. In other words, this detection process prepares the ion in the excited state. Suppose now that at $t=0$ the ion is prepared in its electronic ground state whereas the vibrational motion is in a generic superposition of two-mode Fock states. Equation (64) suggests a simple protocol to generate a prefixed bimodal Fock state $\left|m_{x}, m_{y}\right\rangle$ provided that the probability of finding at $t=0$ the center of mass motion in the state $\left|m_{x}, m_{y}\right\rangle$ is different from zero. In order to generate this state, we imagine to measure several times the internal electronic state at time instants $t_{i}$ appropriately chosen.

The first measurement is performed at the time $t_{1}$ which satisfies the condition $\Omega\left(m_{x}, m_{y}\right) t_{1}=\left(2 l_{1}+1\right) \pi / 2,\left(l_{1}=0,1,2, \cdots\right)$. If the null fluorescence is detected, according to equation (64), the vibrational state $\left|m_{x}, m_{y}\right\rangle$ keeps the original probability unchanged whereas the other components present at $t=0$ decrease their probabilities. In other words, after the renormalization of the state, the probability of the state $\left|m_{x}, m_{y}\right\rangle$ is increased with respect to the other two-mode Fock states. This process can be obviously iterated measuring the null fluorescence at time instants $t_{k}(k=2,3, \cdots)$ defined by the condition 
$\Omega\left(m_{x}, m_{y}\right) t_{k}=l_{k} \pi,\left(l_{k}=1,2,3, \cdots\right)$. It is easy to convince oneself that, following this procedure, the prefixed state $\left|m_{x}, m_{y}\right\rangle$ can be generated. Remembering the Fock state expansion of the pair coherent states, given by equation (59), and noting that all the states $|n+q, n\rangle$ share, in the system under scrutiny, the same Rabi frequency $\Omega\left(m_{x}=n+q, m_{y}=n\right)$, it is possible to persuade oneself that the procedure described before may be used in our context to engineer pair coherent states. It turns out that, starting from a bimodal coherent state $|\alpha, \beta\rangle$ it is possible to generate the pair coherent state $|\xi=\alpha \beta, q\rangle$ with fairly good efficiency. As an example, Fig.11 shows the probability distribution relative to the vibrational state $|\alpha \beta, 2\rangle$ in correspondence to $|\alpha|^{2}=2.5$ and $|\beta|^{2}=1.5$, and $\chi /\left(\Omega_{L x}-\Omega_{L y}\right)=0.0049$. It is worth stressing that this quantum state manipulation procedure requires several successful null fluorescence detections in order to generate the desired state. In the case of the example of Fig.11 the probability of success is $P=0.172$, which is already a significant value from an experimental point of view.

\subsubsection{Schrödinger cat-like states}

Since the famous cat thought experiment suggested by Schrödinger in 1935 [153], superpositions of macroscopically distinguishable quantum states, also known as Schrödinger cat states, have become a longstanding example of the peculiarities which occur in the interpretation of quantum reality. To explore and gain insight into the fundamental issues of quantum theory, a number of schemes have been proposed for the realization of such states. However, the generation of cat states and the detection of their quantum properties is an extremely hard tool since decoherence effects due to the interaction with the external environment tend to transform these quantum superpositions into statistical mixtures destroying their coherence properties. For this reason single trapped ions have shown to be very well suited to the generation of Schrödinger cat states, since, as we have already pointed out, dissipative effects which are inevitable from cavity damping in the optical or microwave regime, can be significantly suppressed for the ion motion due to the extremely weak coupling between the vibrational modes and the external environment. The archetype of a Schrödinger cat states is given by the superposition

$$
|\Psi\rangle_{c a t}=N\left(|\alpha\rangle+e^{i \phi}|-\alpha\rangle\right)
$$

where $\alpha$ is a coherent state of a single mode quantized field and $N$ is a normalization constant. These states are referred to as even, odd and Yurke-Stoler coherent states when $\phi=0, \pi$ and $\pi / 2$ respectively 154 . Schrödinger cat states of this type have been recently realized for a trapped ${ }^{9} B e^{+}$ion 120 . With an average of about nine vibrational quanta, wave packets of maximum separation of about $83 \mathrm{~nm}$, significantly larger than the size of a single component wave packet, about $7 \mathrm{~nm}$, were obtained.

a) Bimodal Schrödinger cat states

In this subsection we review some proposals for generating Schrödinger catlike states of the bidimensional oscillatory motion of a single trapped ion. The first generation scheme we consider 152 allows to engineer cat-like states which 
are the generalization to two modes of motion of the states experimentally realized in 120 . The system considered is an isotropically confined ion irradiated by laser beams in a configuration similar to the one described in the previous paragraph and realizing the effective Hamiltonian (63). The only difference is that here the Rabi frequencies of the two lasers are assumed to be equal $\Omega_{L x}=\Omega_{L y}=\Omega$. With this restriction, the interaction Hamiltonian in the interaction picture assumes the form

$$
\hat{H}_{i n t}^{I}=-\chi\left(\hat{n}_{x}-\hat{n}_{y}\right)\left(\hat{\sigma}_{+}+\hat{\sigma_{-}}\right)
$$

As shown in [152], if the initial state is a bimodal coherent states $|\alpha\rangle|\beta\rangle \equiv|\alpha, \beta\rangle$ and the ion is in its ground internal state, after a time $t>0$ the state of the system evolves to

$$
|\Psi(t)\rangle=|-\rangle\left|S_{+}\right\rangle+|+\rangle\left|S_{-}\right\rangle
$$

where

$$
\left|S_{ \pm}\right\rangle=\frac{1}{2}\left(\left|\alpha e^{i \phi / 2}, \beta e^{-i \phi / 2}\right\rangle \pm\left|\alpha e^{-i \phi / 2}, \beta e^{i \phi / 2}\right\rangle\right)
$$

with $\phi=2 \chi t$. The states $\left|S_{ \pm}\right\rangle$are examples of two-modes cat states of the even and odd type and may be characterized by oscillatory distribution of the vibrational quanta and by strong nonclassical correlations between the modes. It is important to underline the possibility of detecting the entanglement between the modes, which is reflected in the interference between the two components of $\left|S_{+}\right\rangle$. This can be experimentally realized in the same way as in the one mode case, that is by measuring the probability $P_{-}(\phi)$ of finding the ion in its internal ground state. Indeed $P_{-}(\phi)$ is given as (with $\alpha$ and $\beta$ real for simplicity)

$$
P_{-}(\phi)=\left\langle S_{+} \mid S_{+}\right\rangle=\frac{1}{2}\left\{1+e^{-\left(\alpha^{2}+\beta^{2}\right)(1-\cos (\phi))} \cos \left[\left(\alpha^{2}-\beta^{2}\right) \sin \phi\right]\right\}
$$

and is sensitive both to the sum and to the difference of the squares of the amplitudes of the coherent states.

b) Mesoscopic superpositions of pair coherent states

Till now we have been dealing with quite direct generalization of the one mode cat state (65) to two dimensions. There has been however much interest also in the superpositions of macroscopically distinguishable bimodal states such as pair coherent states, SU(2) coherent states, squeezed states and so on. These superpositions are usually referred to as Schrödinger cat-like states. Gou, Steinbach and Knight [155] present a scheme for generating mesoscopic superpositions of two pair coherent states separated in phase by $\phi$ :

$$
|\xi ; q, \phi\rangle=N_{\phi}\left(|\xi ; q\rangle+e^{i \phi}|-\xi ; q\rangle\right)
$$

In equation (70) $N_{\phi}$ is a normalization constant and the pair coherent states $|\xi ; q\rangle$ are defined by equation (59). It is easy to verify that the states $|\xi ; q, \phi\rangle$ are eigenstates of the operator $\left(\hat{a}_{x} \hat{a}_{y}\right)^{2}$ with eigenvalue $\xi^{2}$. The scheme for generating such states is a generalization of the one proposed by the same authors for engineering pair coherent states and discussed in the previous paragraph. Also in this case the desired target state is obtained as steady state solution of 
a master equation describing the effect of spontaneous emissions on a system represented in terms of the following effective interaction Hamiltonian in the interaction picture:

$$
\hat{H}_{i n t}^{I}=\hbar \Omega\left[\left(\hat{a}_{x} \hat{a}_{y}\right)^{2}-\xi^{2}\right] \hat{\sigma}_{-}+h . a .
$$

For general initial states of motion the oscillatory steady state, detected by the extinction of the fluorescence emitted by the ion, is a statistical mixture. However, the authors show that, if the initial state of motion of the ion is prepared in the Fock state $|q, 0\rangle(|q+1,1\rangle)$, then the vibrational steady state is described by an even (odd) pair coherent state.

c) Mesoscopic superpositions of $S U(2)$ coherent states

Let us now consider some recent proposals for generating Schrödinger cat like states based on conditional measurements of the internal state of the ion. The first scheme we wish to describe is aimed at creating superpositions of two $S U(2)$ coherent states separated in phase by $\pi$ 129, 156. The system considered has been already described in subsection III B 3. We have there seen that a single bidimensionally and isotropically confined ion, driven by an appropriate configuration of laser beams (see equation(51)) and prepared in an initial vibrational Fock state along the direction $\bar{x}$ with an angle $\pi / 4$ relative to the $x$ axis, exhibits a temporal behaviour strongly sensitive to the parity of the initial total number of vibrational quanta. It is shown in 129 that, if the ion is initially prepared in the vibrational state $\left|n_{\bar{x}}=N, n_{\bar{y}}=0\right\rangle$ and in its electronic ground state, then the system dynamics, governed by the Hamiltonian (51), is characterized by the existence of instants of time at which the conditional measurement of the electronic ground state projects the c.m. motion into a superposition of two macroscopically distinguishable $\mathrm{SU}(2)$ coherent states. In more detail, if the initial total number of vibrational quanta $N \gg 1$ is even, then at $t=t_{e} / 2=\pi N / 4 \Omega^{\prime}$ the following superposition is generated

$$
\left|\psi\left(t_{e} / 2\right)\right\rangle=\frac{1}{\sqrt{2}}\left(|\tau=1, j=N / 2\rangle+(-1)^{\frac{N}{2}}|\tau=-1, j=N / 2\rangle\right)
$$

whereas, if $N$ is odd, the state generated at $t=t_{o} \simeq t_{e} / 2$ is

$$
\left|\psi\left(t_{o}\right)\right\rangle=\frac{1}{\sqrt{2}}\left(|\tau=i, j=N / 2\rangle-i(-1)^{\frac{N+1}{2}}|\tau=-i, j=N / 2\rangle\right)
$$

Equations (72) and (73) show that the properties of the state generated following the procedure envisaged in 129, strongly depend on the parity of the total initial number $N$ of vibrational quanta. In other words this means that, for even $N$, the state $\left|\psi\left(\frac{t_{e}}{2}\right)\right\rangle$, defined by equation (72), has the form of an even (odd) $\mathrm{SU}(2)$ coherent state 157, if $\frac{N}{2}$ is even (odd). On the other hand, for odd $N$, the two states $\left|\psi\left(t_{o}\right)\right\rangle$, obtained measuring at $t=t_{o}$ the internal state of the ion as $|-\rangle$, may be called SU(2) Yurke-Stoler like coherent states, with a difference of $\frac{\pi}{2}\left(\frac{3 \pi}{2}\right)$ in the relative quantum phase, when $\frac{N+1}{2}$ is even (odd).

It is interesting to look at the states $(72)$ and $(73)$ under another point of view 128 . 
The angular momentum $\hat{L}_{z}=2 \hbar \hat{J}_{2}$ can be express in terms of the right and left circular quanta operators, $\hat{n}_{r}=\hat{a}_{r}^{\dagger} \hat{a}_{r}$ and $\hat{n}_{l}=\hat{a}_{l}^{\dagger} \hat{a}_{l}$ respectively, as follows 158

$$
L_{z}=\hbar\left(\hat{a}_{r}^{\dagger} \hat{a}_{r}-\hat{a}_{l}^{\dagger} \hat{a}_{l}\right)
$$

It is not difficult to verify that the eigenstates $\left|n_{l}=N, n_{r}=0\right\rangle$ and $\mid n_{l}=0, n_{r}=$ $N\rangle$ of $\hat{L}_{z}$, corresponding to its minimum $(-\hbar N)$ and maximum $(+\hbar N)$ eigenvalues respectively, coincide with the $\mathrm{SU}(2)$ coherent states $|\tau=i, j=N / 2\rangle$ and $|\tau=-i, j=N / 2\rangle$. Thus, the two contributing terms in the quantum superposition (73) are eigenstates of the orbital angular momentum correspondent to its minimum and maximum eigenvalue. In Fig.12 (a) we report the spatial distribution of the vibrational state (73) generated with the help of our scheme. This plot clearly shows interference effects which represent a signature of the quantum nature of this superposition. Note that the spatial interference fringes are sensitive to vibrational decoherence [159] and can, thus, be used to study the decoherence induced transition of the quantum superpositions $(73)$ into the correspondent statistical mixture whose spatial distribution is shown in Fig.13 (b). The vibrational state (72), generated in case of even initial $N$, is on the contrary, a superposition of two states describing ionic oscillations along the two orthogonal directions $\bar{x}$ and $\bar{y}$ respectively.

e) $1 D$ Schrödinger cats of bidimensionally confined ions

We conclude this section considering an example of the procedure for generating $1 \mathrm{D}$ cat states exploiting laser beams along the $x-y$ plane. Usually in these schemes the ion is supposed to be confined in a highly anisotropic twodimensional trap. To generate a given state along the $x$ axis of the trap, it is assumed that the ion is tightly bound in the $y$ direction and that the laser along this axis is tuned to the atomic transition. The proposal we wish to review [160] deals with the generation of even and odd squeezed states of the form:

$$
\left|\phi_{ \pm}\right\rangle \propto(|\alpha, \xi\rangle \pm|-\alpha, \xi\rangle)
$$

where $|\alpha, \xi\rangle$ is the squeezed state defined by $|\alpha, \xi\rangle=D(\alpha) S(\xi)|0\rangle$, with $D(\alpha)=$ $\exp \left(\alpha \hat{a}^{\dagger}-\alpha^{*} \hat{a}\right)$ and $S(\xi)=\exp \left(\xi \hat{a}^{\dagger 2}-\xi^{*} \hat{a}^{2}\right)$ displacement and "squeeze" operators respectively. Cat like states as the one of equation (3.3.3) may be engineered as dark state solutions in presence of dissipation due to spontaneous emission. Indeed it is not difficult to prove that even and odd squeezed states satisfy an eigenvalue equation of the form

$$
\left(-g_{1} e^{-i \phi_{1}} \hat{a}_{x}^{2}-g_{2} e^{-i \phi_{2}} \hat{a}_{x}^{\dagger 2}-2 g_{0} e^{-i \phi_{0}} \hat{a}_{x}^{\dagger} \hat{a}_{x}\right) \hat{\rho}=\zeta \hat{\rho}
$$

In order to ensure that the steady state solution effectively approaches a even or odd squeezed state of the form (3.3.3), however, we need to assume that the initial state is prepared with a precise parity [160]. For example starting from the Fock state $|0\rangle$ or $|1\rangle$ one obtains even or odd squeezed states respectively.

\section{Conclusive remarks}

Hamiltonian models describing the coupling between pseudo-spin and bosonic dynamical variables are ubiquitous in physics. The reason is that when one is 
faced with a complex problem like the interaction of a material subsystem (atom, molecule, crystal, etc.) with the radiation field (electromagnetic or elastic), such models catch the essential ingredients of the physical problem and often are exactly solvable. In this paper we have dealt with a special class of such models, namely those describing the coupling of a few-level atom with a pair of bosonic modes. We have shown that these models are not oversimplified representations of given physical situations only, but that, in fact, most of them have been realized in the CQED and trapped ion experimental realms. Our review discusses a wild collection of results achieved over the last few years in both contexts elucidating that their occurrence is chiefly due to the entanglement which gets established in the tripartite system. In particular the possibility of inducing entanglement dependent quantum correlation effects in the two modes system is shown. This very attractive aspect as well as the generation of many nonclassical bimodal states, clearly witness the dynamical richness of the hamiltonian models which we have focused on in this review paper.

\section{Acknowledgements}

The authors acknowledge F.S. Persico for useful suggestions and for reading part of the manuscript.

\section{References}

[1] Rempe G.,Schmidt-Kaler, F., and Walther, H., 1990, Phys. Rev. Lett., 64, 2783 ;

[2] Krause, J., Scully, M. O., and Walther, H., 1987, Phys. Rev. A, 36, 4547; Meystre, P., Zubairy, M.S., 1982, Phys. Lett., 89 A, 390; Rempe, G., Walther, H., and Klein, N., 1987, Phys. Rev. Lett., 58, 353;

[3] Haroche, S., 1992, in Fundamental Systems in Quantum optics, (North Holland, Amsterdam);

[4] Walther, H., 1994, in Advances in Atomic, Molecular and Optical Physics, vol. 32, 379 (Academic Press);

[5] Brune, M., Schmidt-Kaler, F., Maali, A., Dreyer, J., Hagley, E., Raimond, J. M., and Haroche, S., 1996, Phys. Rev. Lett., 76, 1800;

[6] Jaynes, E. T., Cummings, F. W., 1963, Proc. IEEE, 51, 89;

[7] Shore, B. W., and Knight, P. L., 1993, J. Mod. Opt., 40, 1195;

[8] Meschede, D., Walther, H., and Muller, G., 1985, Phys. Rev. Lett., 54, 551;

[9] Buck, B., Sukumar, C. V., 1981, Phys. Lett. A, 81, 132;

[10] Gerry, C. C., 1988, Phys. Rev. A, 37, 2683;

[11] Ashraf, I., Gea-Banacloche, J., and Zubairy, M. S., 1990, Phys. Rev. A, 42, 6704; 
[12] Knight, P. L., 1986, Physica Scripta, 33, 51;

[13] Phoenix, S. J. D., Knight, P.L., 1990, J. Opt. Soc. Am. B, 7, 116;

[14] Buzek, V., Jex, I., 1989, Quant. Opt., 2, 147;

[15] Benivegna, G. B., Messina, A, Napoli, A., 1994, Phys. Lett. A, 194, 353;

[16] Quang, T., Knight, P. L., and Buzek, V., 1991, Phys. Rev. A, 44, 6092;

[17] Barnett, S. M., and Knight, P. L., 1986, Phys. Rev. A, 33, 2444;

[18] Tavis, M., Cummings, F. W., 1969, Phys. Rev., 188, 1132;

[19] Haroche, S., and Raimond, J. M., 1985, Adv. atom. molec. Phys., 20, 347;

[20] Knight, P.L., and Quang, T., 1990, Phys. Rev. A, 41, 6255;

[21] Buck, B., and Sukumar, C. V., 1984, J. Phys. A, 17, 877;

[22] Kozierowski, M., and Shumovsky, A. S., 1987, Physica A, 145, 290;

[23] Parker, J., and Stroud, C. R., 1986, J. Opt. Soc. Am. B, 3, 96;

[24] Benivegna, G. B., Messina, A., 1988, Phys. Rev. A, 37, 4747;

[25] Brune, M., Raimond, J. M., and Haroche, S., 1987, Phys. Rev. A, 35, 154; Davidovich, L., Raimond, J. M., Brune, M., and Haroche, S., 1987, Phys. Rev. A, 36, 3771;

[26] Maia-Nieto, P. A., Davidovich, L., Raimond, J. M., 1991, Phys. Rev. A, 43, 5073;

[27] Manzoor, I., Zhu, S.Y, and Zubairy, S., 2000, Opt. Comm. 184, 417; Napoli, A., Messina, A., and Maniscalco, S., 2001, in Quantum Communication, Computing and Measurement 3, P. Tombesi, O. Hirota Eds., (Kluwer Academic/Plenum Publisher ), 415; Napoli, A., Messina, A., Maniscalco, S., 2000, Acta Physica Slovaca 50, 519.

[28] Davidovich, L., Zagury, N. , Brune, M., Raimond, J.M., and Haroche, S., 1994, Phys. Rev A, 50, R895;

[29] Zubairy, M. S., 1998, Phys. Rev. A, 58, 4368;

[30] Cohen-Tannoudji, C., Dupon-Roc, J., and Grynberg, G., 1992, Atomphoton interactions, (J. Wiley and Sons, New York); Allen, L., and Eberly, J. H., 1975, Optical resonance and two level atoms, (New York);

[31] Eberly, J.H., Naroznhy, N.B., Sanchez-Mondragon, J.J., 1980, Phys. Rev. Lett., 44, 1323; Naroznhy, N.B., Sanchez-Mondragon, J.J., Eberly, J.H., 1987, Phys. Rev. A., 23, 236;

[32] Shore, B. W., 1979, Am. J. Phys., 47, 262;

[33] Alsing, P. and Zubairy, M.S., 1987, J. Opt. Soc. Am. B, 4, 177;

[34] Puri R. R., and Bullough, R.K., 1998, J. Opt. Soc. Am B, 5, 2021; 
[35] Gerry, C. C., and Eberly, J. H., 1990, Phys. Rev. A , 42, 6805;

[36] Joshi, A., and Puri R. R., 1990, Phys. Rev. A , 42, 4336;

[37] Cardimona D. A., Kovanis, V., Sharma, M. P., and Gavrielides, A., 1991, Phys. Rev. A, 43, 3710;

[38] Brune, M., Raimond, J. M., Goy, P., Davidovich, L., and Haroche, S., 1987, Phys. Rev. Lett. , 59, 1899;

[39] Xiao-shen Li, and Lin, D.L., 1987, Phys. Rev. A, 36, 5209;

[40] Zhen-don Liu, Xiao-shen Li, and Lin, D.L., 1987, Phys. Rev. A, 36, 5220;

[41] Yoo, H.I., and Eberly, J.H., 1985, Phys. Rep., 118, 239;

[42] Lai, W. K., Buzek, V., and Knight, P.L., 1991, Phys. Rev A, 44, 2003;

[43] Buzek, V., and Quang, T., 1989, J. Opt. Soc. Am. B , 6, 2447 and references therein;

[44] Zheng-dong Liu, Liu D. L., and Thomas, F. G., 1991, Phys. Rev. A, 44, 6144

[45] Mahran, M. H., 1990, Phys. Rev. A, 42, 4199;

[46] Loudon, R., and Knight, P. L., 1987, J. Mod. Opt., 34, 709;

[47] Hillery, M., 1989, Phys. Rev. A, 40, 3147;

[48] Poizat, J. Ph., Collett, M. J., and Walls, D. F., 1992, Phys. Rev. A, 45, 5171

[49] Ho Trung Dung, Tanas, R., and Shumovsky, A. S., 1991, Quantum Opt., 3, 255 ;

[50] Pegg, D. T., Barnett, S. M., 1988, Europhys. Lett., 6, 483;

[51] Arun, R., Agarwal, G. S., Scully, M. O., and Walther, H., 2000, Phys. Rev. $A, \mathbf{6 2}, 023809$;

[52] Scully, M.O., Walther, H., Agarwal, G. S., Tran Quang, and Schleich, W., 1991, Phys. Rev. A, 44, 5992;

[53] Benivegna, G.B., and Messina, A., 1994, J. Mod. Opt., 41, 907;

[54] Benivegna, G. B., 1996, J. Mod. Opt., 43, 1589;

[55] Laughlin, D. W., and Swain, S., 1991, Quantum Opt., 3, 77;

[56] Deb, B., Gangopadhyay, G., and Deb Shankar Ray, 1993, Phys. Rev. A, 48, 1400;

[57] Gangopadhyay, G., Ray, D. S., 1992, Phys. Rev. A, 45, 1843;

[58] Gerry, C. C., and Huang, H., 1992, Phys. Rev. A, 45, 8037;

[59] Mahran, M. H., 1992, Phys. Rev. A, 45, 5113; 
[60] Lai ,W. K., Buzek, V., Knight, P. L., 1991, Phys. Rev. A, 44, 6043;

[61] Cardimona, D.A., Vassilios Kovanis, and Sharma, M. P., 1993, Phys. Rev. $A, 47,1227$

[62] Ying Wu and Xiaoxue Yang, 2001, Phys. Rew. A, 63, 043816-1;

[63] Napoli, A., Messina, A., 1996, J. Mod. Opt., 43, 649;

[64] Gou, S.-C., 1989, Phys. Rev. A, 40, 5116;

[65] Gou, S.-C., 1990, Phys. Lett. A, 147, 218;

[66] Joshi, A., Puri, R. R., 1990, Phys. Rev. A, 42,4336;

[67] Gerry, C. C., Welch, R. F., 1992, J. Opt. Soc. Am. B, 9, 290;

[68] Ashraf, M., 1994, Phys. Rev. A, 50, 5116;

[69] Napoli, A., Messina, A., 1997, Quantum and Semiclass. Opt., 9, 587;

[70] Napoli, A., Messina, A., 1999, in Mysteries, Puzzles and Paradoxes in Quantum Mechanics, Bonifacio, R., ed. (American Institute of Physics, Woodbury, N.Y. ) p.331;

[71] Napoli, A., Messina, A., 2000, in Nuclear and Condensed Matter Physics, Messina, A., ed. (American Institute of Physics), p.186;

[72] Braginski, V. B., and Kalhili F. Ya., 1995, Quantum Measurement, (Cambridge University Press, Cambridge)

[73] Ekert, E., Josza, R., 1996, Review of Mod. Phys. 68, 733.

[74] For a recent review see the book D. Bouwmeester, A. Ekert and A. Zeilinger The physics of quantum information, 2000 (Springer-Verlag) and references therein.

[75] Bennet, C., Di Vincenzo, D.P., 2000, Nature, 404, 247.

[76] Ekert, A.K., 1991, Phys. Rev. Lett., 67, 661.

[77] Krause, J., Scully, M. O., Walther, T., and Walther, H., 1989, Phys. Rev. A, 39, 1915;

[78] Garraway, B. M., Sherman, B., Moya-Cessa, H., Knight, P. L., and Kurizki, G., 1994, Phys. Rev. A, 49, 535;

[79] Gerry, C. C., 1996, Phys. Rev. A, 54, R2529;

[80] Harel G., Kurizki, G., 1996, Phys. Rev. A, 54, 5410;

[81] Davidovich, L., Brune, M., Raimond, J. M., and Haroche, S., 1996, Phys. Rev. A, 53, 1295;

[82] Napoli, A., Messina, A., 1997, J. Mod. Opt. , 44, 2093;

[83] Napoli, A., Messina, A., 1997, J. Mod. Opt., 44, 2075; 
[84] Haroche S., 1998, Physica Scripta, T76, 159;

[85] Napoli, A., 1998, Phys Rev. A , 58, 2528;

[86] Weidinger, M., Varcoe, B. T. H., Heerlein, R., and Walther, H., 1999, Phys. Rev. Lett., 82, 3795;

[87] Englert, B.-G., and Walther, H., 2000, Opt. Comm., 179, 283;

[88] Raushenbeutel, A., Bertet, P., Osnaghi, S., Nogues, G., Brune, M., Raimond, J. M., and Haroche, S., 2001, Phys. Rev. A, 64, 050301;

[89] Brune, M., Hagley, E., Dreyer, J., Matre, X., Maali, A., Wunderlich, C., Raimond, J. M., and Haroche, S., 1996, Phys. Rev. Lett., 77, 4887;

[90] Zurek, W., H., 1991, Physics Today, 44, 36;

[91] Deb, B., Gangopadhyay, G., and Deb Shankar Ray, 1995, Phys. Rev. A, $\mathbf{5 1}, 2651$;

[92] Vogel, K., 1993, Phys. Rev. Lett., 71, 1816;

[93] Zheng, S.-B., Guo, G.-C., 1997, Quantum and Semiclassical Opt., 9, 1041;

[94] Guo, G.-C., Zheng, S.-B., 1997, Opt. Comm., 133, 142;

[95] Caves, C. M., Drummond, P. D., 1994, Rev. Mod. Phys., 66, 481;

[96] Napoli, A., Messina, A., 2000, J.Mod. Opt., 47, 2105;

[97] Napoli, A., Messina, A., 1998, Opt. Comm., 156, 32;

[98] Napoli, A., Messina, A., 2000, J. Opt. B: Quantum and Semiclass. Opt., 2, 282;

[99] Napoli, A., Messina, A., 1999, Acta Physica Slovaca, 49, 783;

[100] Wineland, D.,and Dehmelt, H. G., 1975, Bull. Am. Phys. Soc., 20, 637; Neuhauser, W., Hohenstatt, M., Toschek, P. E., and Dehmelt, H. G., 1978, Phys. Rev. Lett., 41, 233; Wineland, D., Drullinger, R., and Walls, F., 1978, Phys. Rev. Lett., 40, 1639; Neuhauser, W., Hohenstatt, M., Toschek, P. E., and Dehmelt, H. G., 1980, Phys. Rev. A, 22, 1137; Diedrich, F., Bergquist, J. C., Itano, W. M., and Wineland, D. J. 1989, Phys. Rev. Lett., 62, 403; Monroe, C., Meekhof, D. M., King, B. E., Jefferts, S. R., Itano, W. M., and Wineland, D. J., 1995, Phys. Rev. Lett., 75, 4011.

[101] For reviews on cooling and trapping see, for example: Toschek, P. E., 1984, Atomic Particles in trap, in New Trends in Atomic Physics Vol I, Proceedings of the Les Houches Summer School, Session XXXVIII, edited by G. Grynberg and R. Stora, (Amsterdam: North Holland), pp.382-450; Blatt, R., 1992, Laser cooling of trapped ions, in Fundamental Systems in Quantum Optics, Proceedings of the Les Houches Summer School, Session LIII, edited by J. Dalibard, J. M. Raymond and J. Zinn-Justen and R. Stora, (New York: Elsevier), pp.255-286; Stenholm, S., (1986), Rev. Mod. Phys., 58, 699. 
[102] Blockley, C. A., Walls, D. F., and Risken, H., 1992, Europhys. Lett., 17, 509; Blockley, C. A., and Walls, D. F., 1993, Phys. Rev. A, 47, 2115

[103] Cirac, J. I., Blatt, R., Parkins, A. S., and Zoller, P., 1994, Phys. Rev. A, 491202

[104] Meekhof, D. M., Monroe, C., King, B.E., Itano, W. M., and Wineland, D. J., 1996, Phys. Rev. Lett., 76, 1796; Leibfried, D., Meekhof, D. M., Monroe, C., King, B.E., Itano, W. M., and Wineland, D. J., 1997, J. Mod. Opt., 44, 2485

[105] Brune, M., Schmidt-Kaler, F., Maali, A., Dreyer, J., Raimond, J.M., and Haroche, S., 1996, Phys. Rev. Lett., 76, 1800

[106] Roos, Ch., Zeiger, Th., Rohde, H., Nägerl, H. C., Eschner, J., Leibfried, D., Schmidt-Kaler, F., and Blatt, R., 1999, Phys. Rev. Lett., 83, 4713; Schmidt-Kaler, F., Roos, Ch., Nägerl, H. C., Rohde, H., Gulde, S., Mundt, A., Lederbauer, M., Thalhammer, G., Zeiger, Th., Barton, P., Hornekaer, L., Reymond, G., Leibfried, D., Eschner, J., and Blatt, R., 2000, J. Mod. Opt., 47, 2573

[107] Feynman, R., 1982, Int. J. Theor. Phys., 21, 467; Feynman, R., 1985, Opt. News, 11, 11

[108] Wallentowitz, S., and Vogel, W., 1997, Phys. Rev. A, 55, 4438

[109] Wallentowitz, S., Vogel W., and Knight, P. L., 1999, Phys. Rev. A, 59, 531

[110] Agarwal, G. S., and Banerji, J., 1997, Phys. Rev. A, 55, R4007

[111] Gou, S.-C., and Knight, P. L. 1996, Phys. Rev. A, 54, 1682

[112] Wineland, D. J., Monroe, C., Itano, W. M., Leibfried, D., King, B. E., and Meekhof, D. M., 1998, J. Res. Natl. Inst. Stand. Technol., 103, 259

[113] Wineland, D. J., Monroe, C., Itano, W. M., King, B. E., Leibfried, D., Myatt, C., and Wood, C., 1998, Physica Scripta, T76, 147

[114] Vogel, W., and de Matos Filho, R.L., 1995, Phys. Rev. A, 52, 4214

[115] de Matos Filho, R. L., and Vogel, W. , 1998, Phys. Rev. A, 5b, R1661

[116] Wallentowitz, and S., Vogel W., 1998, Phys. Rev. A, 58, 679

[117] Paul, W., 1990 Rev. Mod. Phys., 62, 531; Horvath, G. Zs. K., Thompson, R. C., and Knight, P. L., 1997, Contemp. Phys., 38, 25.

[118] Ghosh, P. K., 1995, Ion traps, (Oxford: Clarendon Press), pp.382-450

[119] Vogel, W., and Wallentowitz, S., 2001, Manipulation of the quantum state of a trapped ion, in Coherence and Statistic of photons and atoms, edited by J. Peřina, (New York: Wiley)

[120] Monroe, C., Meekhof, D. M., King, B. E., and Wineland D. J.,1996 Science, 272, 1131 
[121] Leibfried, D., Meekhof, D. M., King, B.E., Monroe, C., Itano, W. M., and Wineland, D. J., 1996, Phys. Rev. Lett., 77, 4281

[122] Nagourney, W., Sandberg, J., and Dehmelt, H., 1986, Phys. Rev. Lett., 56, 2797; Bergquist, J. C., Hulet, R. G., Itano, W. M., and Wineland D. J., 1986, Phys. Rev. Lett., 57, 1699; Sauter, Th., Neuhauser, W., Blatt, R., and Toschek, P. E., 1986, Phys. Rev. Lett., 57, 1696.

[123] Drobný, G., and Hladký, B., 1997, Acta Phys. Slov., 47, 277.

[124] Steinbach, J., Twamley, J., and Knight, P. L., 1997, Phys. Rev. A, 56, 4815

[125] Holland, M. J., Burnett, K., 1993, Phys. Rev. Lett., 71, 1355; Kim, T., Pfister, O., Holland, M. J., Noh, J., and Hall, J. L., 1998, Phys. Rev. A, $\mathbf{5 7}, 4004$

[126] Maniscalco, S., Messina, A., and Napoli, A., 2000, Acta Phys. Slov., 50, 333

[127] Maniscalco, S., Messina, A., and Napoli, A., 2001, J. Mod. Opt., in press

[128] Maniscalco, S., Messina, A., and Napoli, A., 2000, J. Mod. Opt., 47, 2113

[129] Maniscalco, S., Messina, A., and Napoli, A., 2000, Phys. Rev. A, 61, 053806

[130] Maniscalco, S., Messina, A., and Napoli, A., 2001, Rep. on Math. Phys, in press

[131] Maniscalco, S., Messina, A., Napoli, A., and Vitali, D., 2001, J. Opt. B: Quantum and Semiclass. Opt., accepted for pubblication

[132] Maniscalco, S., Messina, A., Napoli, A., and Vitali, D., 2001,in Quantum Communication, Computing and Measurement 3, edited by P. Tombesi and O. Hirota, (New York: Kluwer Academic/Plenum Publishers), pp. 419-422

[133] Gardiner, S. A., Cirac, J. I., and Zoller, P., 1997, Phys. Rev. A, 55, 1683

[134] Law, C. K., and Eberly, J. H., 1996, Phys. Rev. Lett., 76, 1055

[135] Kneer, B., and Law, C. K., 1998, Phys. Rev. A, 57, 2096

[136] Greenberger, D. M., Horne, M. A., Shimony, A. and Zeilinger, A., 1990,Am. J. Phys., 58, 1131; Greenberger, D. M., Horne, M. A., and Zeilinger, A., 1993,Phys. Today, 46(8), 22

[137] Drobný, G., and Hladký, B., and Bužek, 1998,Phys. Rev. A, 58, 2481

[138] Zheng, S.-G., 2001,Phys. Rev. A, 63, 015801

[139] Morigi, G., Cirac, J. I., Ellinger, K., and Zoller, P., 1998, Phys. Rev. A, $\mathbf{5 7}, 2909$

[140] Hladký, B., Drobný, B., and Bužek, 2000, Phys. Rev. A, 61, 022102 
[141] Perelemov, A. M., 1972 Comm. Math. Phys., 26, 222; Gilmore, R. 1972 Ann. Phys., 74, 391; Zhang, W., Feng, D. H., and Gilmor, R., 1990 Rev. Mod. Phys., 62, 867; Perelemov, A. M., 1985, Generalized Coherent States and their Application, (Berlin: Springer-Verlag).

[142] Campos, R. A., Saleh, B. E. A., and Teich, M. C., 1989, Phys. Rev. A, 40, 1371

[143] Kis, Z., Vogel, W., Davidovich, L. and Zagury, N., 2001, Phys. Rev. A, 63, 053410

[144] Gou, S.-C., Steinbach, J., and Knight, P. L., 1996, Phys. Rev. A, 54, R1014

[145] Gerry, C. C., Gou, S.-C.,and Steinbach, J., 1997, Phys. Rev. A, 55, 630

[146] Agarwal, G. S., 1988, J. Opt. Soc. Am. B, 5, 1940; Agarwal, G. S., 1986, Phys. Rev. Lett., 57, 827.

[147] Vanden Berghe, G., and De Meyer, H. 1978, J. Phys. A, 11, 1569

[148] Merzbacher F., 1970, Quantum Mechanics, (New York: Wiley), pp. 156160

[149] Wang, K., Maniscalco, S., Messina, A., and Napoli, A., 2001, Laser Phys., 11, 476

[150] Wang, K., Maniscalco, S., Messina, A., and Napoli, A., 2001, Chin. Phys. Lett., in press

[151] Wang, K., Maniscalco, S., Messina, A., and Napoli, 2001, Phys. Rev. A, 63, 043419

[152] Gerry, C. C., 1997, Phys. Rev. A, 55, 2478

[153] Schroedinger, E., 1935, Naturwissenschaften, 23, 807

[154] Yurke, B., and Stoler, D., 1986, Phys. Rev. Lett., 57, 13

[155] Gou, S.-C., Steinbach, J., and Knight, P. L., 1996, Phys. Rev. A, 54, 4315

[156] Maniscalco, S., Messina, A., and Napoli, A. 1999,in Nuclear and Condensed Matter Physics, AIP Conference Proceeding, edited by A. Messina, (New York: American Institute of Physics ), pp. 154

[157] Gerry, C. C., and Grobe, R., 1997, J. Mod. Opt., 44, 41

[158] Cohen-Tannoudji, C., Diu, B., and Laloë, F., 1977,in Quantum Mechanics Vol. I, (New York: John Wiley and Sons ), pp. 727-741.

[159] Zeng, H., 1998, Phys. Rev. A, 57, 388; Zeng, H., Lin, F., Wang, Y., and Segawa, Y., 1999, Phys. Rev. A, 59, 4589

[160] Gou, S.-C., Steinbach, J., and Knight, P. L., 1997, Phys. Rev. A, 55, 3719 
Figure 1: Energy level diagram of a three-level atom (a) in the $\Lambda$ configuration; (b) in the $\Xi$ configuration and (c) in the $V$ configuration.

Figure 2: Average photon number in mode 1 for $g_{1}=g_{2}=g$ and $n=20$

Figure 3: Energy level diagram of a three-level atom in the $\Lambda$ configuration. The detuning $\Delta$ is assumed large enough with respect to $E_{3}-E_{1}$ in order to adiabatically eliminate the level $E_{2}$.

Figure 4: Energy level diagram of a three-level atom interacting with the two modes of the field.

Figure 5: Time evolution of the $\mu=1$ average photon number, for $r_{1}=r_{2}=$ $s=0$, corresponding to the initial condition (a) $n=20$ and (b) $n=21$.

Figure 6: Schematic view of the axial section of a Paul trap

Figure 7: Raman excitation scheme coupling the electronic states $|+\rangle$ and $|-\rangle$ and the vibrational levels through a third upper electronic level $|s\rangle$

Figure 8: Vibrational Raman scheme leaving the electronic state unchanged

Figure 9: Scheme for the measurement of the electronic state of the ion by means of quantum jumps techniques

Figure 10: Time evolution of $\left\langle\hat{J}_{1}(t)\right\rangle$ for initial total number of vibrational quanta $N=20$ (gray line) and $N=21$ (black line)

Figure 11: Probability distributions in the process of generating the pair coherent state $\mid \alpha \beta, q=2>_{p c s}$ from the initial coherent state $\mid \alpha, \beta>$ with $|\alpha|^{2}=2.5$ and $|\beta|^{2}=1.5$. The phase difference of the two driving beams is $\phi=\pi$, and $\chi /\left(\Omega_{L x}-\Omega_{L y}\right)=0.0049$

Figure 12: Spatial distribution of the superposition $\frac{1}{\sqrt{2}}\left(\left|n_{l}=N, n_{r}=0\right\rangle+i\left|n_{l}=0, n_{r}=N\right\rangle\right)$, for $N=21$, against $x^{\prime}=\frac{x}{\beta}$ and $y^{\prime}=\frac{y}{\beta}$ with $\beta=\sqrt{\frac{m \nu}{\hbar}}$ 
Figure 13: Spatial distribution of the statistical mixture of the two states $\mid n_{l}=$ $\left.N, n_{r}=0\right\rangle$ and $\left|n_{l}=0, n_{r}=N\right\rangle$, for $N=21$, against $x^{\prime}=\frac{x}{\beta}$ and $y^{\prime}=\frac{y}{\beta}$ with $\beta=\sqrt{\frac{m \nu}{\hbar}}$ 
(a)

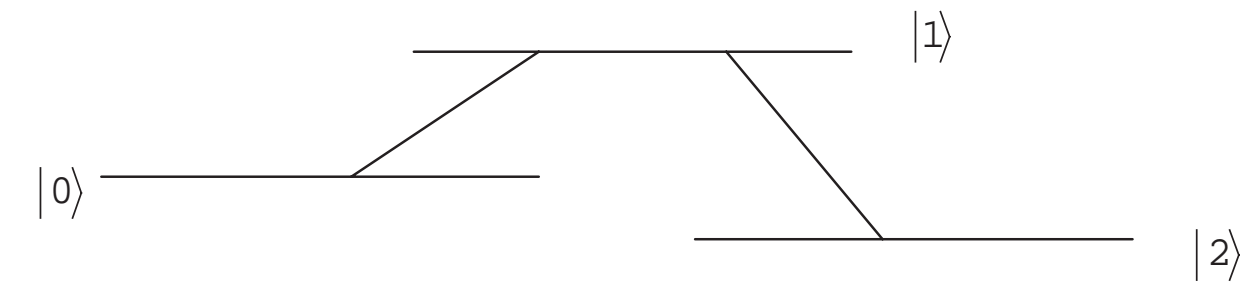

(b)

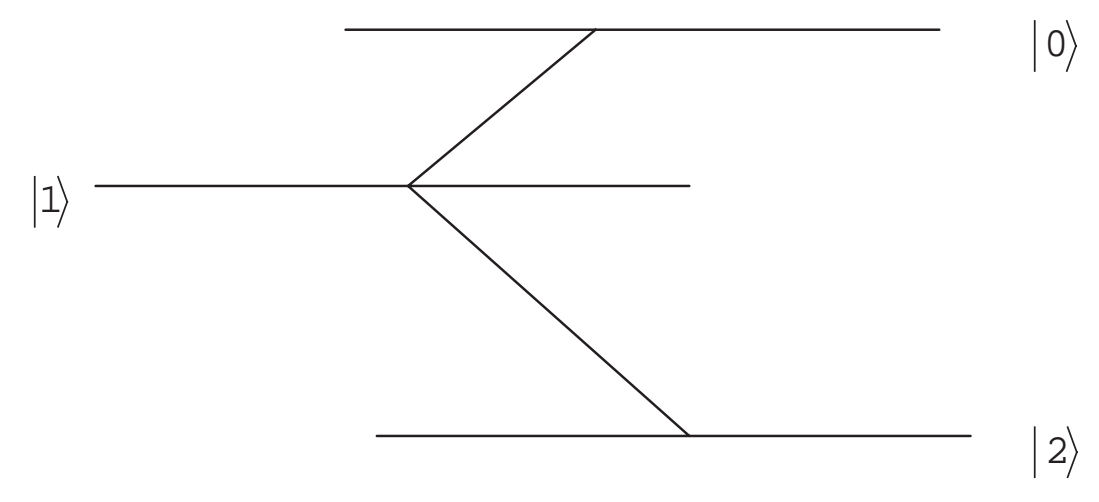

(c)

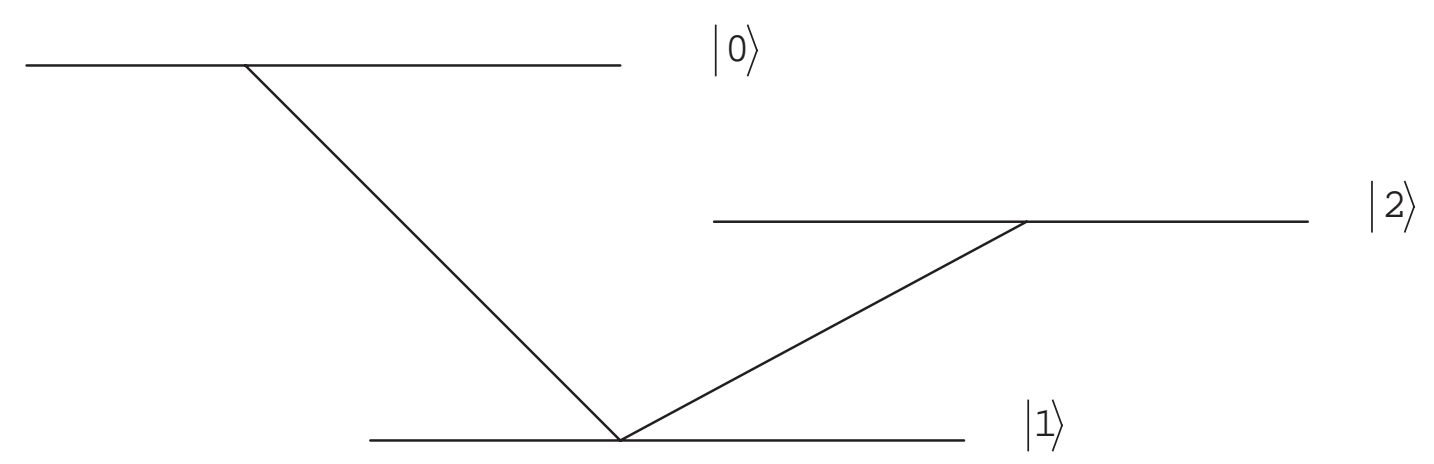


20

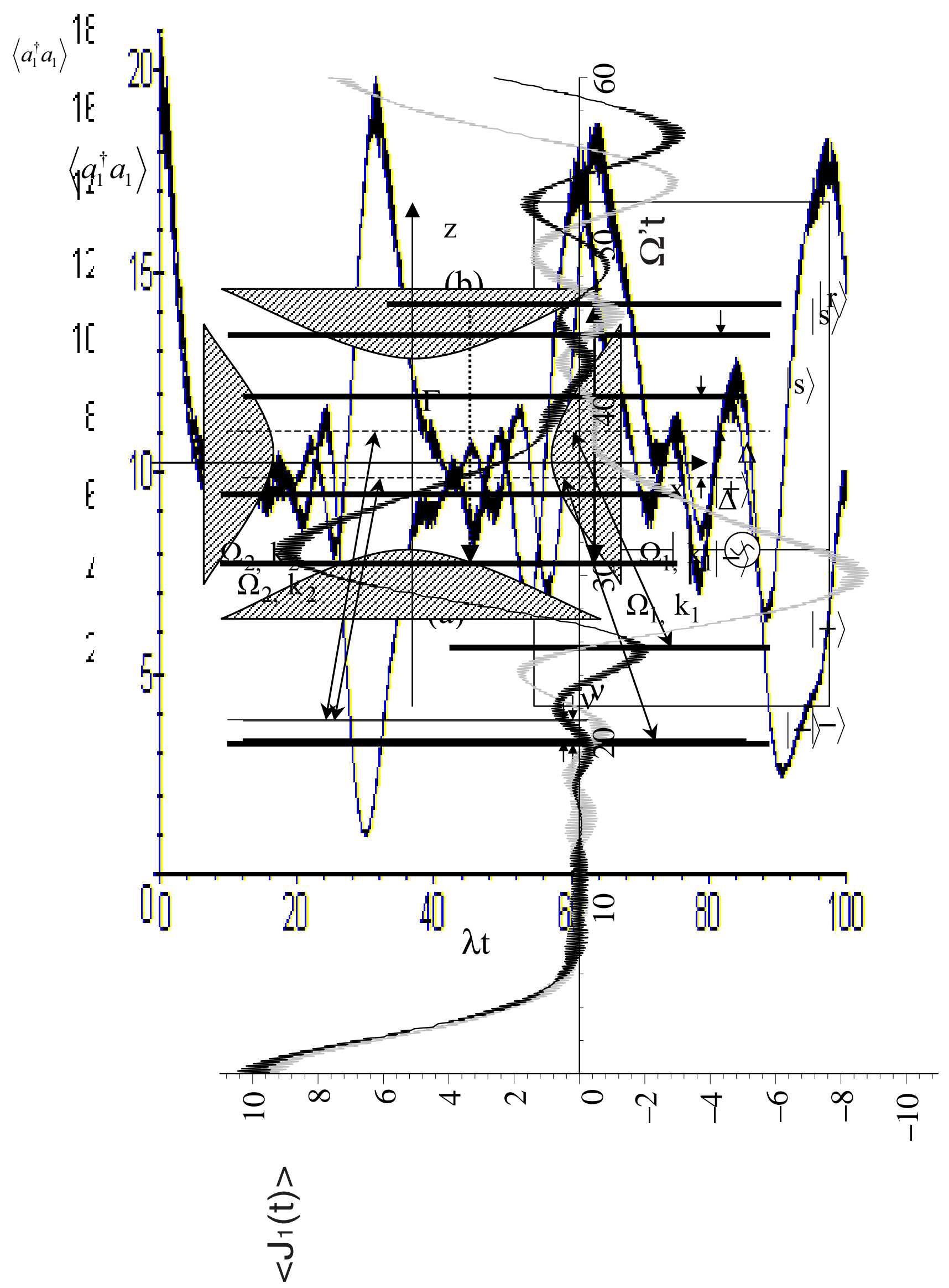




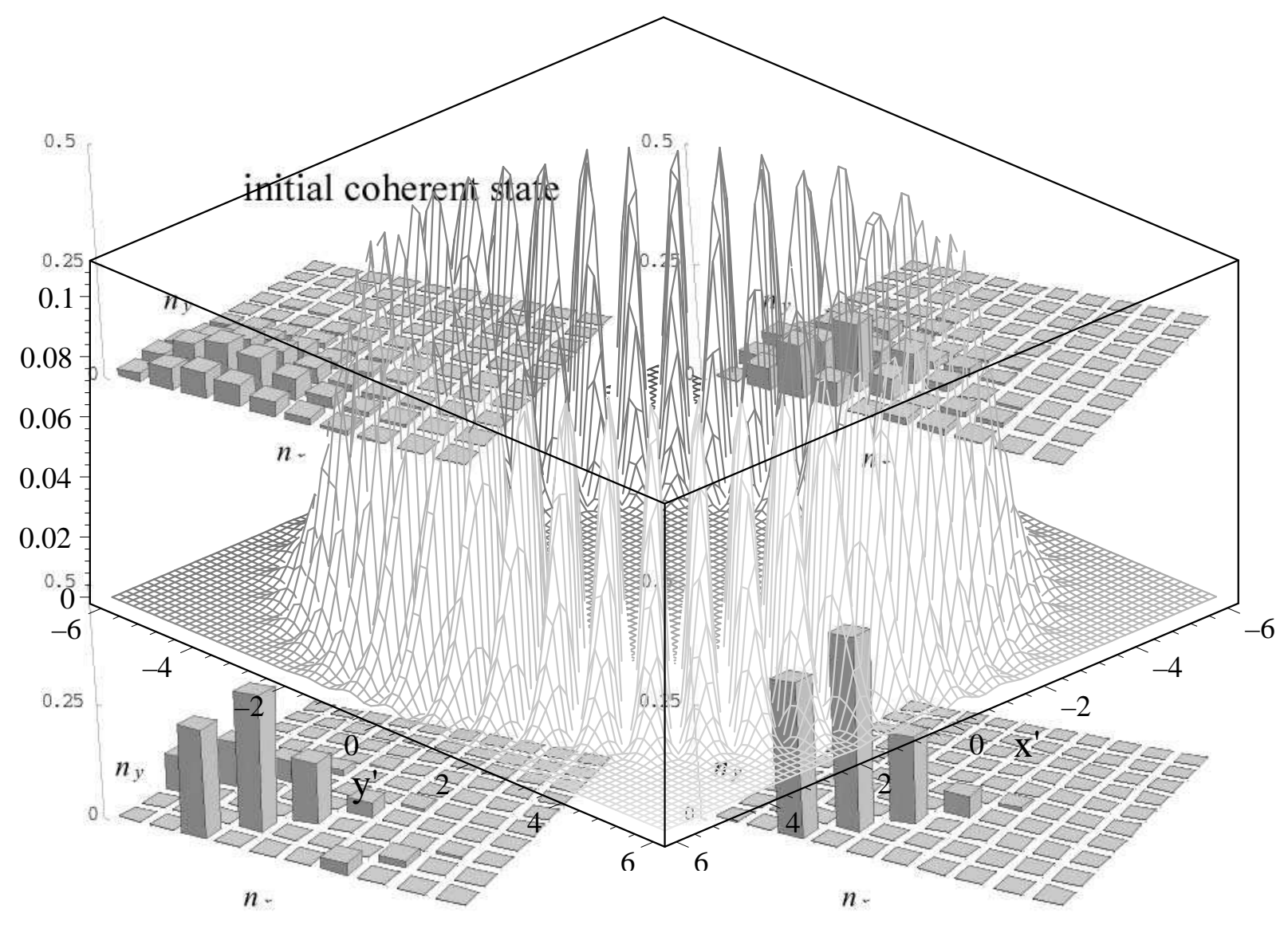




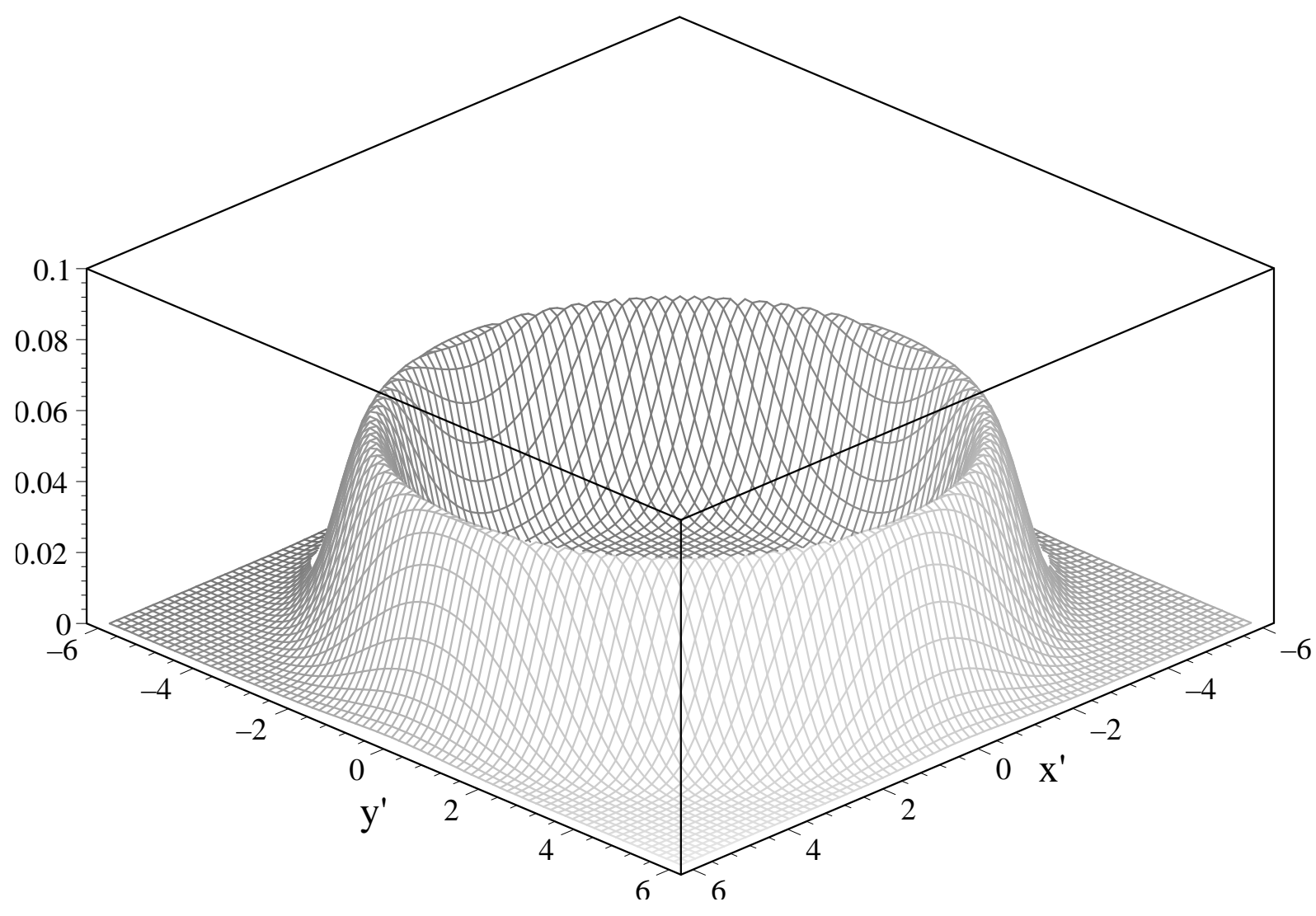

